\title{
Poly(ethylene oxide) Functionalized Graphene Nanoribbons with Excellent Solution Processability
}

\section{Supporting Information}

Yinjuan Huang ${ }^{1}$, Yiyong Mai ${ }^{1}$, Uliana Beser ${ }^{2}$, Joan Teyssandier ${ }^{3}$, Gangamallaiah Velpula $^{3}$, Hans van Gorp ${ }^{3}$, Lasse Arnt Straas $\phi^{4}$, Michael Ryan Hansen ${ }^{5}$, Daniele Rizzo $^{6}$, Cinzia Casiraghi ${ }^{6}$, Rong Yang ${ }^{7}$, Guangyu Zhang ${ }^{7}$, Dongqing Wu ${ }^{1}$, Fan Zhang $^{1}$, Deyue Yan ${ }^{1}$, Steven De Feyter ${ }^{3}$, Klaus Müllen ${ }^{2}$, Xinliang Feng ${ }^{1,8} *$

${ }^{1}$ School of Chemistry and Chemical Engineering, Shanghai Jiao Tong University, 800 Dongchuan RD, Shanghai 200240, China, ${ }^{2}$ Max Planck Institute for Polymer Research, Ackermannweg 10, 55128, Mainz, Germany, ${ }^{3}$ Division of Molecular Imaging and Photonics, Department of Chemistry, KU Leuven Celestijnenlaan, 200 F, B-3001 Leuven, Belgium, ${ }^{4}$ Interdisciplinary Nanoscience Center, Aarhus University, Gustav Wieds Vej 14, DK-8000 Aarhus C, Denmark, ${ }^{5}$ Institute of Physical Chemistry, Westfälische Wilhelms-Universität Münster, Corrensstr. 28/30, D-48149 Münster, Germany, ${ }^{6}$ School of Chemistry, Manchester University, Oxford Road, Manchester M139PL, United Kingdom, ${ }^{7}$ Institute of Physics, Chinese Academy of Sciences, P.O. Box 603, Beijing 100190, China, ${ }^{8}$ Department of Chemistry and Food Chemistry, Technische Universität Dresden, Mommsenstrasse 4, 01062 Dresden, Germany

E-mail: mai@ @stu.edu.cn (Y. M.); xinliang.feng@tu-dresden.de (X. F.)

\section{Materials.}

Materials were purchased from Sigma-Aldrich, Alfa Aesar, Admas-beta, and J\& K suppliers and used as received, unless otherwise mentioned.

\section{Instrumentation and Methods}

Analytical thin layer chromatography (TLC) was performed on silica gel coated substrates "60 F254" from Merck. Solution nuclear magnetic resonance $\left({ }^{1} \mathbf{H}\right.$ NMR and ${ }^{13} \mathbf{C}$ NMR) spectra were recorded on a Mercury Plus 400 (400 MHz for proton, $100 \mathrm{MHz}$ for carbon) spectrometer with tetramethylsilane as the internal reference. Data are reported via chemical shift (ppm, the solvent resonance as the internal standard), integration and multiplicity (Abbreviations: $\mathrm{s}=$ singlet, $\mathrm{d}=$ doublet, 
$\mathrm{t}=$ triplet, $\mathrm{m}=$ multiplet). Matrix-assisted laser desorption/ionization time-of-flight (MALDI-TOF) mass spectra were obtained via a Bruker Reflex II utilizing a $337 \mathrm{~nm}$ nitrogen laser, calibrated against poly(ethylene glycol) (3000 $\mathrm{g} / \mathrm{mol}$ ). Molecular weights of the polymers were obtained by gel permeation chromatography (GPC) with a refractive index detector (Shimadzu RID-10A) using tetrahydrofuran (THF) as the eluent, and the samples were referenced with respect to standard polystyrene (PS) calibration curves. Recycling GPC was conducted on Shodex K-2003 GPC columns using chloroform as eluent. Fourier transform infrared (FTIR) spectra were recorded on a Spectrum 100 (Perkin Elmer, Inc., USA) spectrometer. Thermal gravimetric analysis (TGA) was measured using a TA-Instruments Q5000IR thermo gravimetric analyzer in flowing $(100 \mathrm{ml} / \mathrm{min})$ nitrogen. The tests were performed at an increasing rate of $5{ }^{\circ} \mathrm{C} / \mathrm{min}$. Ultraviolet-Visible (UV-vis) absorption spectra were recorded on a HITACHI U-4100 spectrometer. Fluorescence spectra were collected via a Princeton Instruments Acton 2500i grating spectrometer. Samples were sonicated for 4 hours at a concentration of $0.01 \mathrm{mg} / \mathrm{ml}$ in $\mathrm{THF}$ or $\mathrm{NMP}$ at $\sim 45^{\circ} \mathrm{C}$. Measurements were performed after one hour of resting, by which time the optical density of the dispersion was stable. Transmission electron microscopy (TEM) observations were performed on a JEM-2100 (JEOL Ltd., Japan) with an accelerating voltage of $200 \mathrm{kV}$. TEM samples were obtained by dropping the solutions of the GNR assemblies onto carbon-coated copper grids, followed by vacuum-drying for 6 hours; no staining treatment was performed. Scanning electron microscope (SEM) observations were 
carried out on a FEI Sirion-200 field-emission scanning electron microscope at an electric voltage of $10 \mathrm{KV}$. Atomic force microscope (AFM) measurements of the GNR thin-film devices were performed using a scanning probe microscope (Multimode Nanoscope, USA) operated in tapping mode by using silicon nitride cantilevers with a force constant of $0.12 \mathrm{~N} / \mathrm{m}$. Dynamic Light Scattering (DLS) was performed on a Malvern Zetasizer Nano $\mathrm{S}$ apparatus with a $4.0 \mathrm{~mW}$ laser $(\lambda=633$ $\mathrm{nm})$. The measurements were conducted at 10 or $20^{\circ} \mathrm{C}$ at a scattering angle of $90^{\circ}$. The CONTIN program was used for the processing of the data.

\section{Synthesis of polyphenylene (PP-COOCH 3$)$}

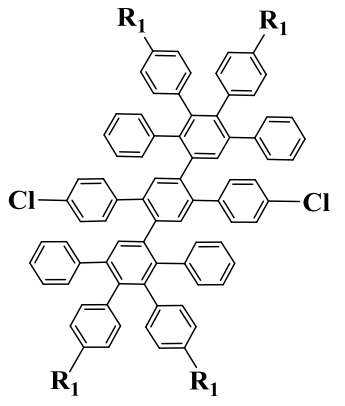

Monomer i) $\operatorname{COD}(\mathrm{Ni}) / \mathrm{COD}, 2,2^{\prime}$-bipyridine DMF/toluene, $80^{\circ} \mathrm{C}, 72 \mathrm{~h}$

$85 \%$

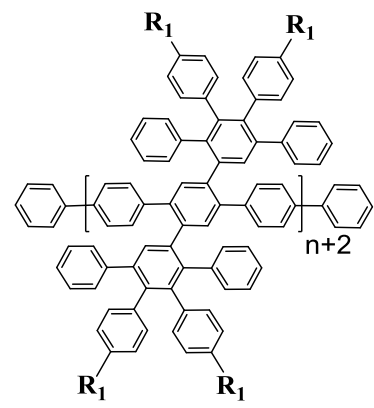

$\mathrm{PP}_{-} \mathrm{COOCH}_{3}$ (PP-1, PP-2, PP-3)

Scheme 1. Synthetic route towards $\mathrm{PP}-\mathrm{COOCH}_{3}$.

The monomer was prepared in our previous work. ${ }^{1,2}$ The polymerization was carried out via Yamamoto reaction. Firstly, a mixture of 2,2'-bipyridine (38 mg, 0.25 mmol), bis(cyclooctadiene)nickel(0) $(72 \mathrm{mg}, 0.25 \mathrm{mmol})$, and cyclooctadiene (0.065 $\mathrm{ml}, 0.25 \mathrm{mmol}$ ) were dissolved in a mixed solvent of dimethyl formamide (DMF) $(0.50 \mathrm{ml})$ and toluene $(2.0 \mathrm{ml})$, and then the mixture was stirred at $60{ }^{\circ} \mathrm{C}$ for $30 \mathrm{~min}$. Secondly, a solution of the monomer (100 mg, $0.054 \mathrm{mmol})$ in $0.5 \mathrm{ml}$ of DMF and 1.0 
$\mathrm{ml}$ of toluene was added. The resultant mixture was stirred at $80{ }^{\circ} \mathrm{C}$ for $72 \mathrm{~h}$ in dark. Finally, $0.5 \mathrm{ml}$ of anhydrous chlorobenzene was added and the mixture was further stirred for $2 \mathrm{~h}$. After reaction, the mixture was cooled down to room temperature and passed through a silica column (eluent: THF) to remove the catalyst. After evaporation of the redundant THF, the residue was dropwise added into methanol, yielding white precipitate. The precipitate was collected by filtration, followed by vacuum drying, producing an off-white solid product $(81 \mathrm{mg}, 85 \%$ yield). The product was further purified by recycling GPC to obtain $\mathrm{PP}-\mathrm{COOCH}_{3}(\mathrm{PP}-1, \mathrm{PP}-2$ and PP-3) with narrow molecular weight distributions. The $\mathrm{PP}-\mathrm{COOCH}_{3}$ samples were employed for the subsequent synthesis of GNR-COOCH 3 (GNR-1, GNR-2 and GNR-3).

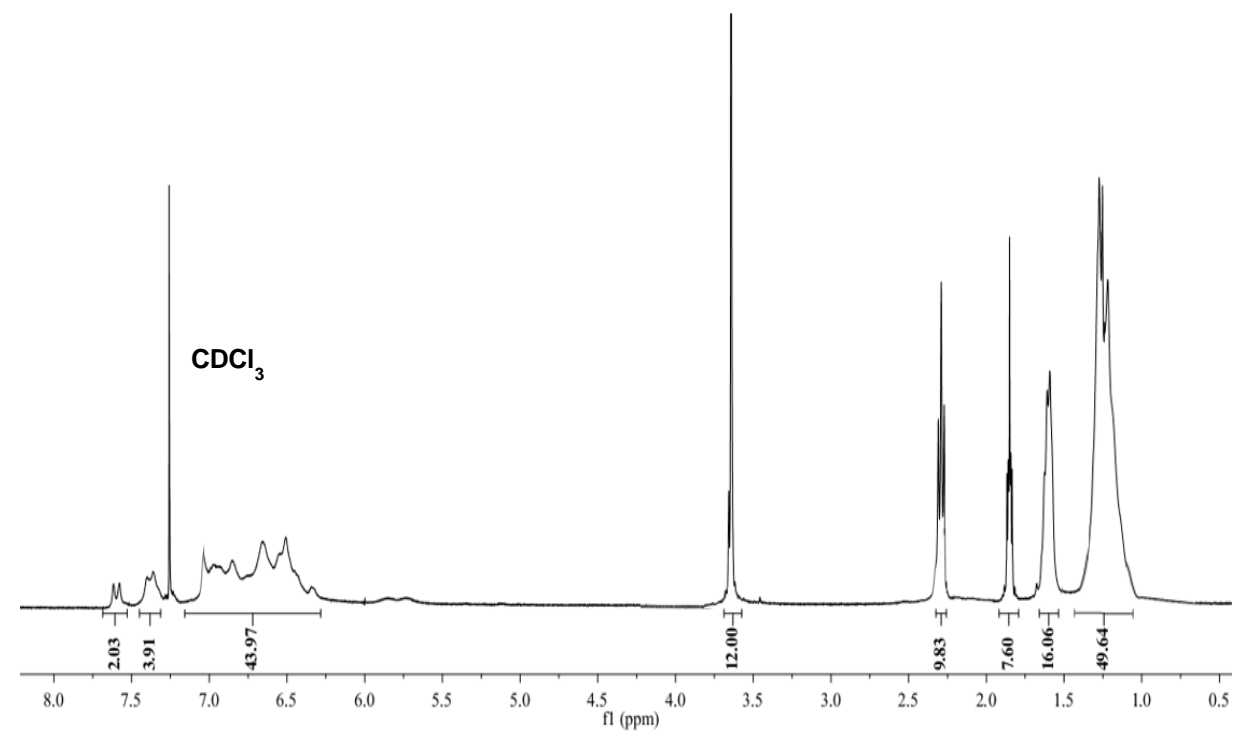

Figure S1. ${ }^{1} \mathrm{H}$ NMR spectrum of $\mathrm{PP}-\mathrm{COOCH}_{3}$. 


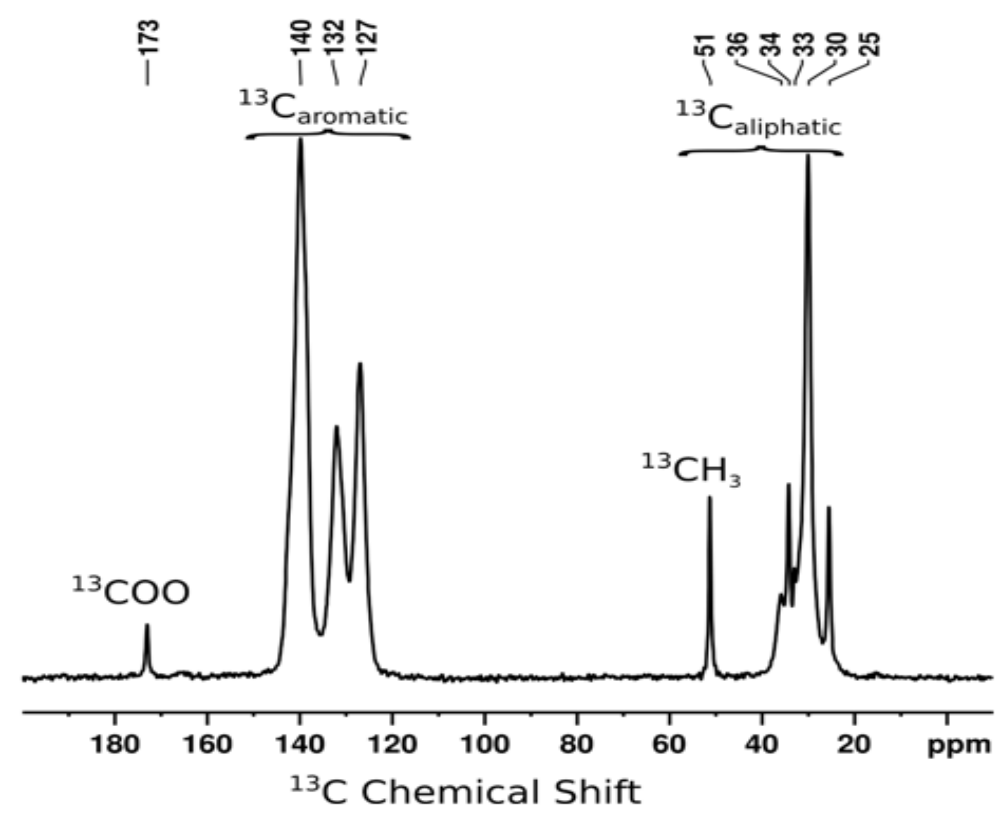

Figure S2. Solid ${ }^{13} \mathrm{C}$ NMR spectrum of $\mathrm{PP}-\mathrm{COOCH}_{3}$.

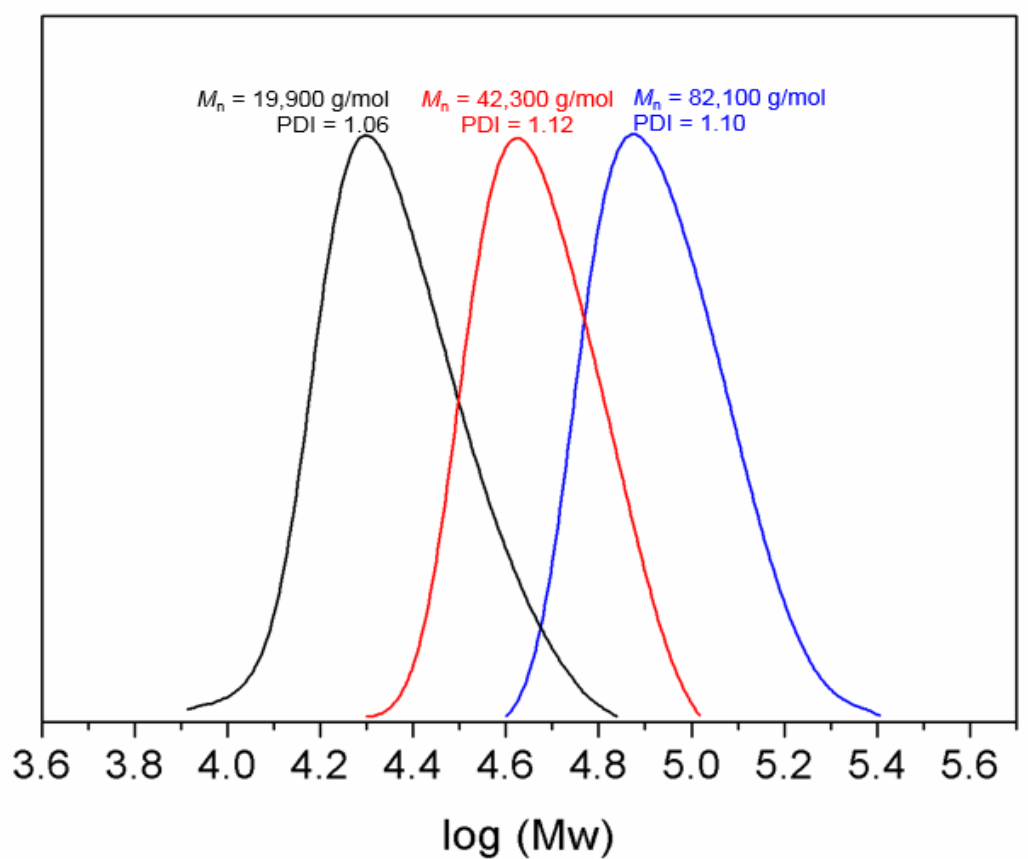

Figure S3. GPC curves of PP-1, PP-2 and PP-3 after purification. Based on the $M_{\mathrm{n}}$, the degrees of polymerization (DP) of the three polyphenylenes are calculated to be $\sim 11,23$ and 44, respectively (THF, PS standard). 


\section{Synthesis of nanographene $(\mathrm{C78})$ from the monomer ${ }^{1}$}

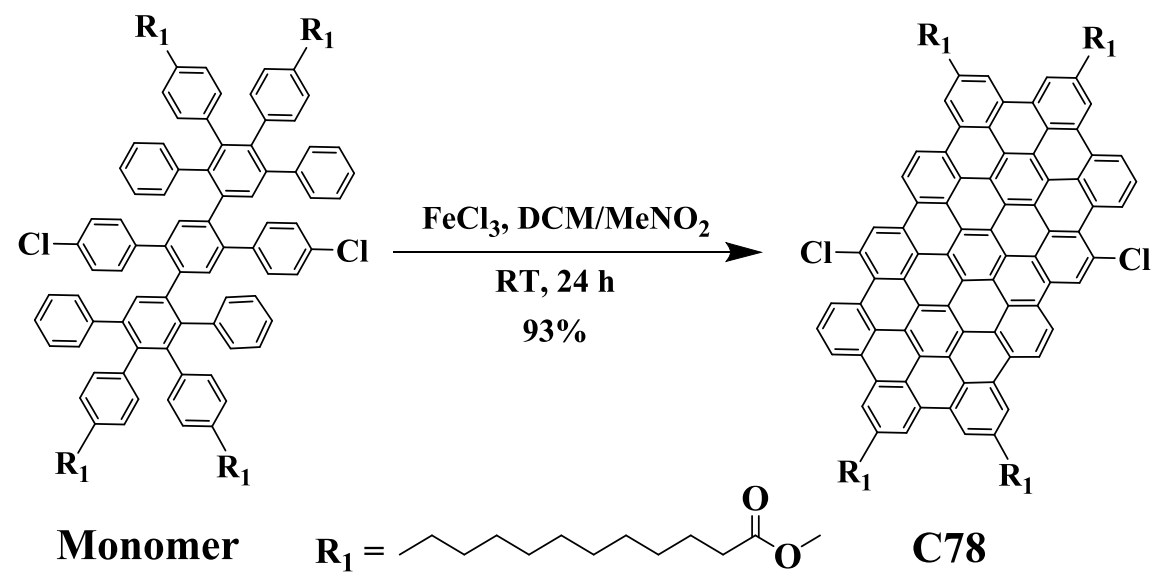

Scheme 2. Synthetic route towards C78.

The monomer $(50.0 \mathrm{mg}, 27.0 \mu \mathrm{mol})$ was dissolved in $50.0 \mathrm{ml}$ of dichloromethane. Followed by the addition of ferric chloride $(920 \mathrm{mg}, 5.66 \mathrm{mmol}, 7.5 \mathrm{eqv} . / \mathrm{H})$ dissolved in $2.0 \mathrm{ml}$ of nitromethane. Then a stream of nitrogen saturated with dichloromethane was passed through the reaction mixture for $2 \mathrm{~h}$. The mixture was stirred at room temperature for $24 \mathrm{~h}$. At last, an excess amount of methanol was added, producing precipitates. The precipitates were collected by extraction filtration and washed with water and methanol for at least 3 circles. After vacuum drying, $46.0 \mathrm{mg}(25.2 \mu \mathrm{mol})$ of reddish brown powder (C78) was obtained, with a yield of $92 \%$.

${ }^{1} \mathrm{H}$ NMR (400 MHz, Dichlorobenzene- $d_{4}$, Figure S4): $\delta$ 7.67-7.65 (s, 4H), 7.40-7.35 (s, 4H), 7.34-7.30 (m, 10H), 7.07-7.04 (s, 2H).

Elemental Analysis: Calculated: C 82.92, H 6.19, Cl 3.88, O 7.01; Found: C 82.16, H $6.45,07.05$.

MS (MALDI-TOF, Figure S5): m/z (\%): 1825 (100), 1823 (75), (calcd. $\mathrm{C}_{126} \mathrm{H}_{112} \mathrm{Br}_{2} \mathrm{Cl}_{8}=1823$ - isotop. distr.: 1824 (100), 1823 (73). 


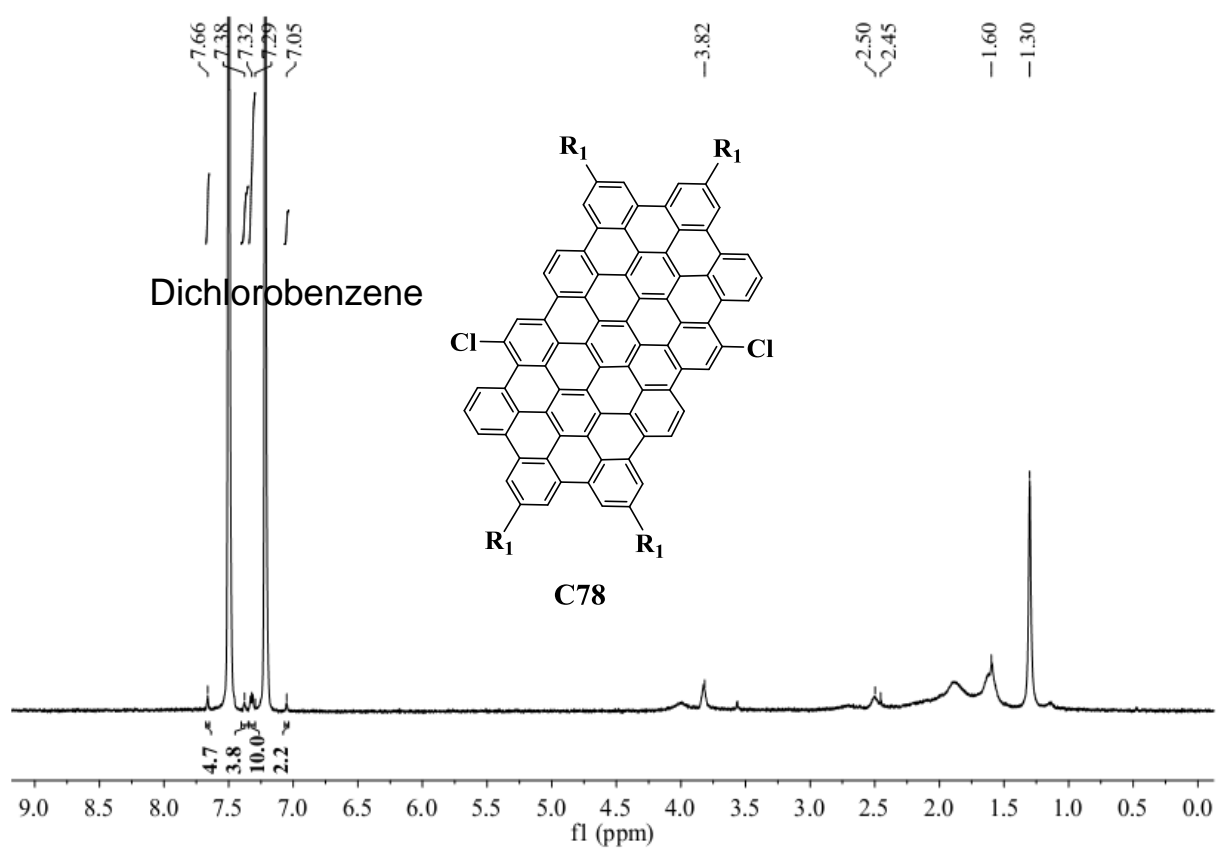

Figure S4. ${ }^{1} \mathrm{H}$ NMR spectrum of C78.

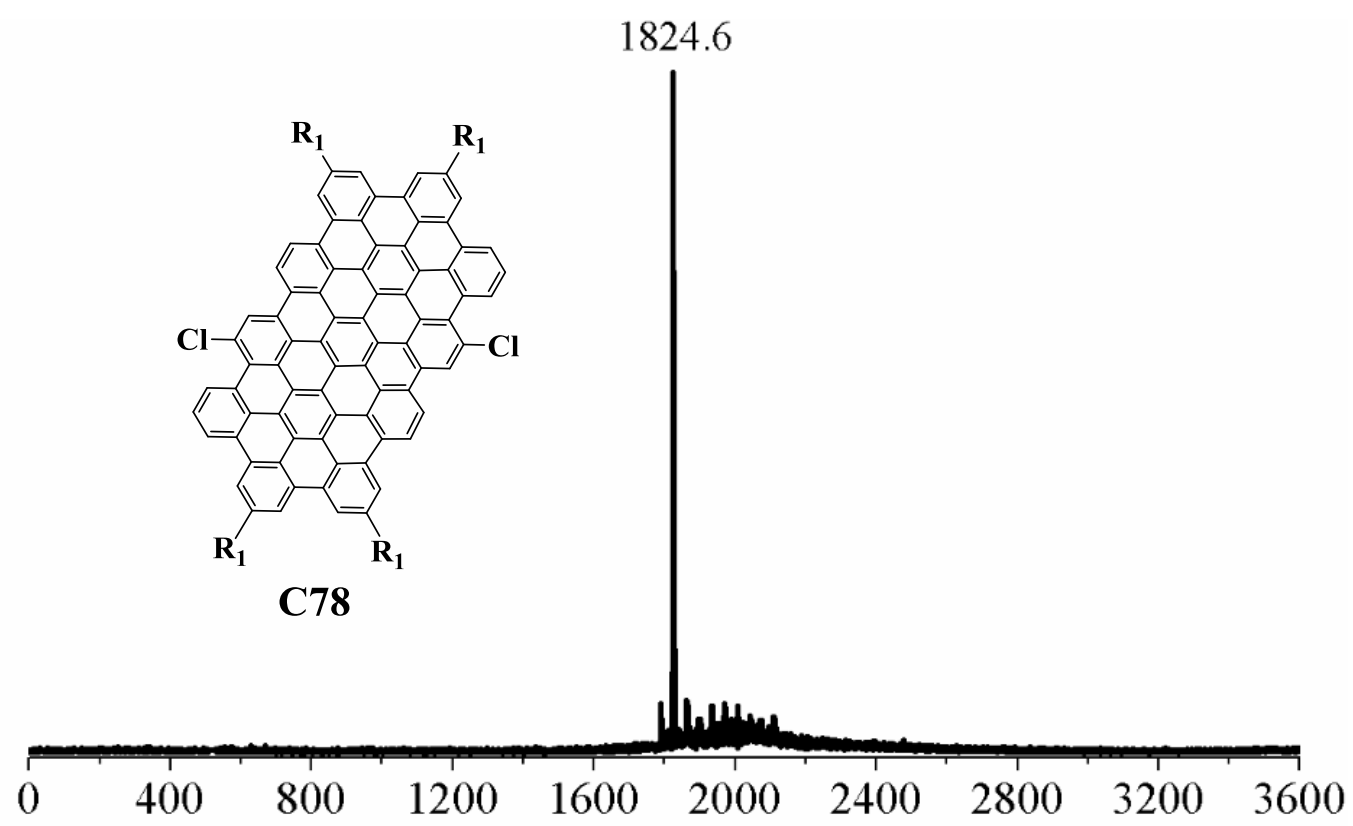

Figure S5. MALDI-TOF MS analysis of C78 (matrix: TCNQ). 


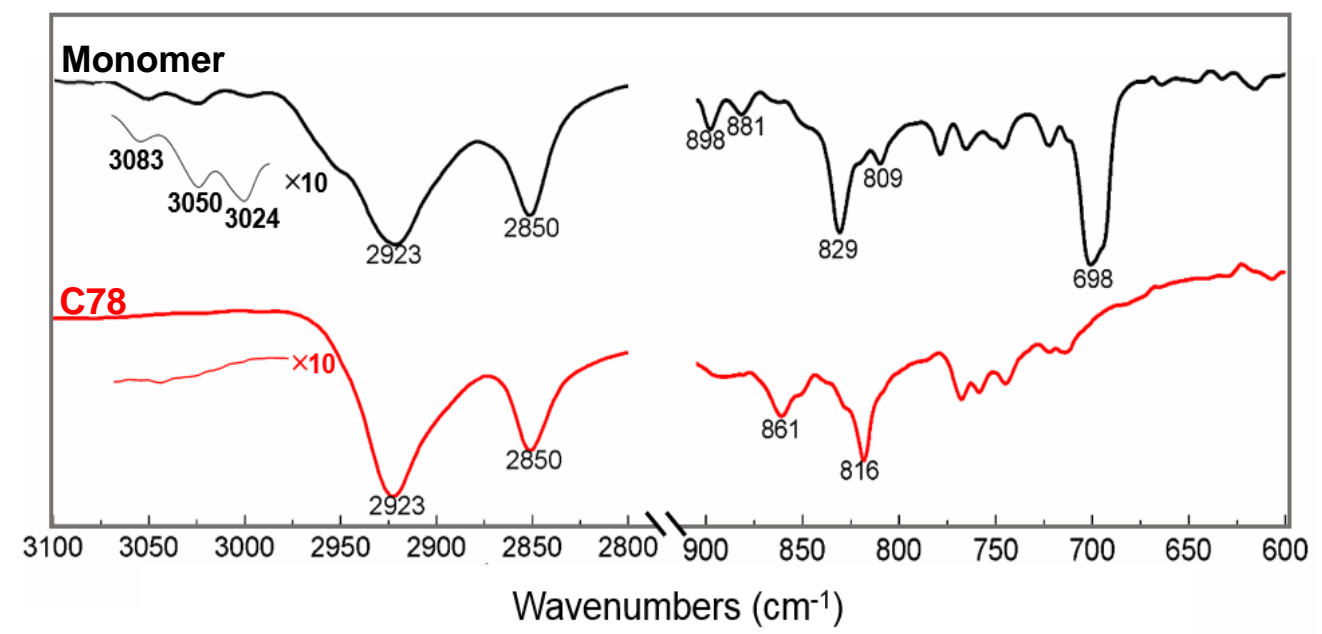

Figure S6. FTIR spectral regions for the monomer and C78 after cyclodehydrogenation. The great attenuation of the signals from aromatic $\mathrm{C}-\mathrm{H}$ stretching vibrations at 3083,3050 and $3024 \mathrm{~cm}^{-1}$ in the spectrum of $\mathrm{C} 78$, the disappearance of fingerprint out-of-plane (opla) $\mathrm{C}-\mathrm{H}$ deformation bands at 829, 809 and $698 \mathrm{~cm}^{-1}$, which originate from mono- and disubstituted benzene rings, and the appearance of the typical opla bands for aromatic $\mathrm{C}-\mathrm{H}$ at the peripheral positions at 816 and $861 \mathrm{~cm}^{-1}$ validate the efficient cyclodehydrogenation of the oligophenylene. , $^{3,4}$

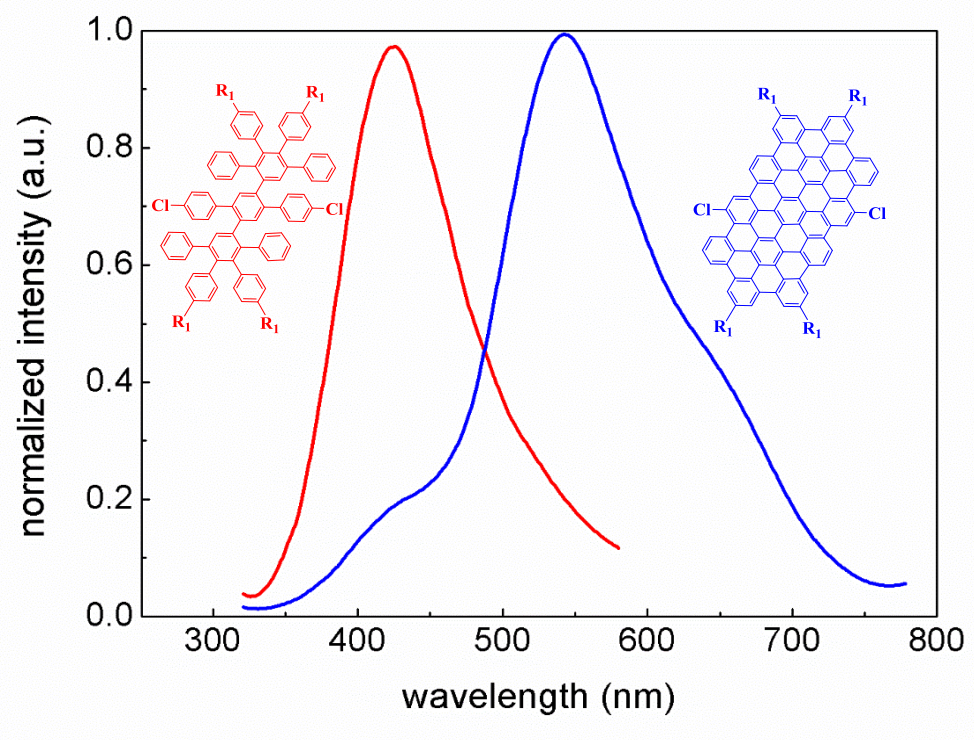

Figure S7. Normalized fluorescence emission spectra of the monomer and C78 in THF with concentration of $1 \times 10^{-4} \mathrm{M}$. 


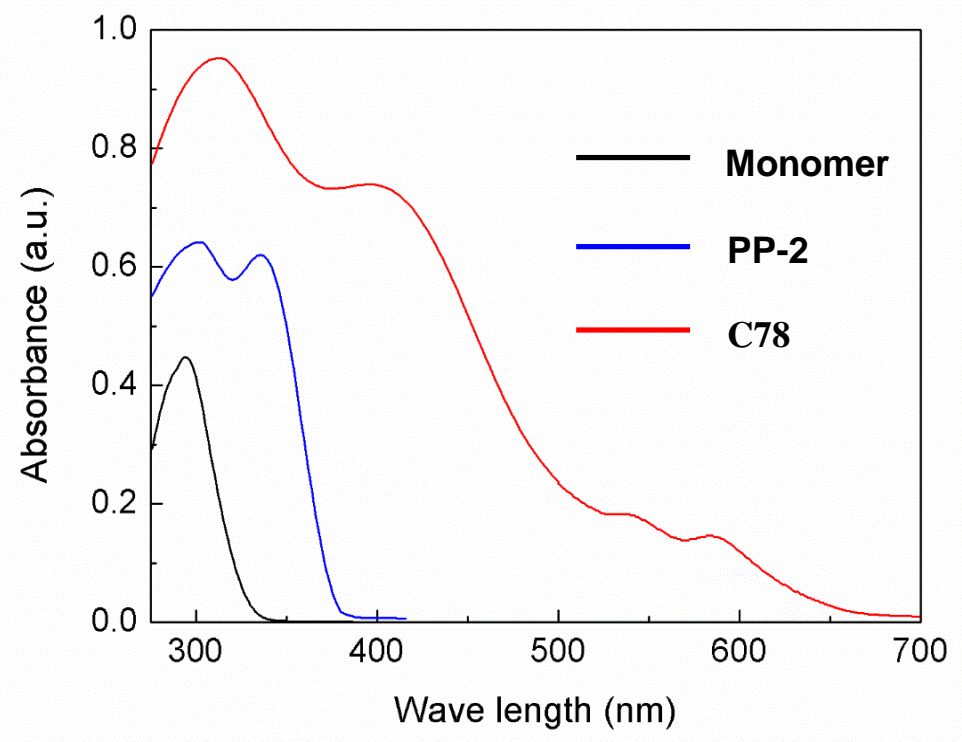

Figure S8. UV-vis spectra of the monomer, C78 and PP-2 in THF (concentration: 0.1 $\mathrm{mg} \mathrm{mL}^{-1}$ ). It is remarkable to note that the monomer shows only one absorption peak at $298 \mathrm{~nm}$, while the spectrum of PP-2 shows two peaks at 298 and $340 \mathrm{~nm}$. For C78, the $\beta$-band is observed at $428 \mathrm{~nm}$ and the $\mathrm{p}$ - and $\alpha$-band appear at 530 and $580 \mathrm{~nm}$, respectively. ${ }^{5}$

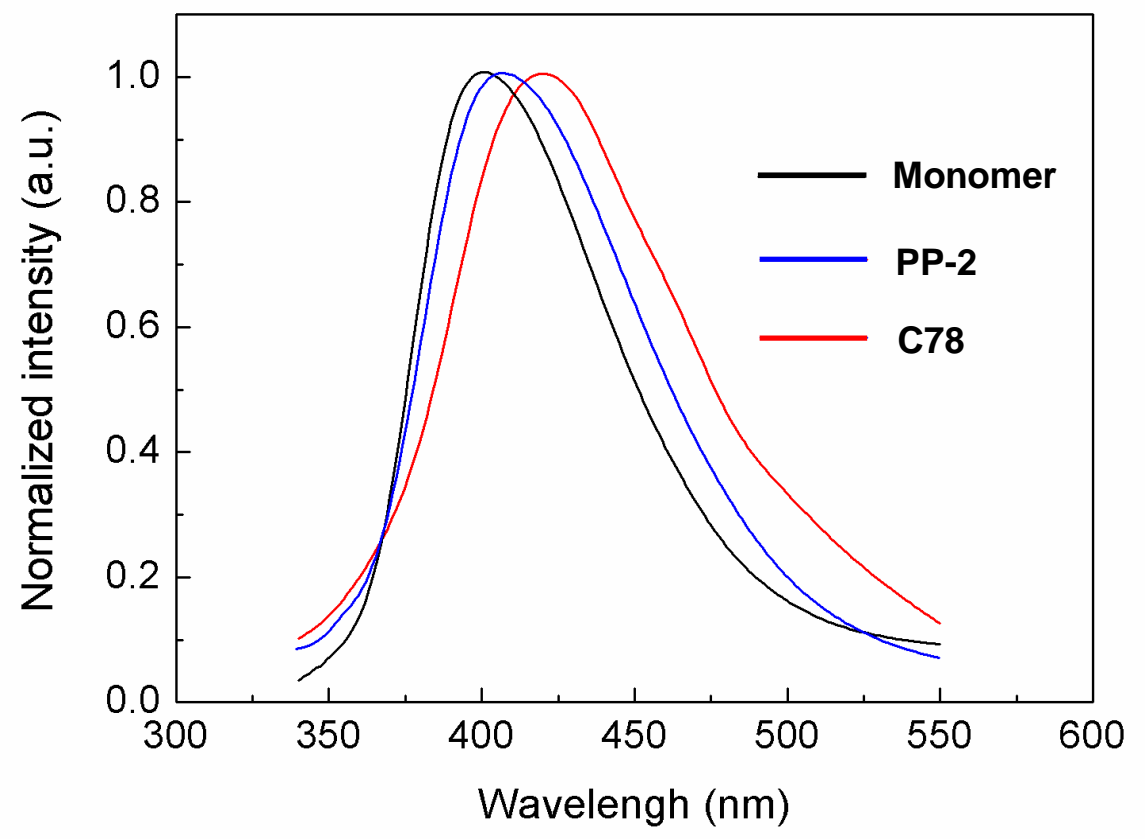

Figure S9. Photoluminescence spectra of the monomer, C78 and PP-2 in THF (concentration: $0.1 \mathrm{mg} \mathrm{mL}^{-1}$ ). 


\section{Synthesis of GNR-COOCH 3 , GNR-COOH, and GNR-PEO.}

\subsection{Synthesis of GNR-COOCH}

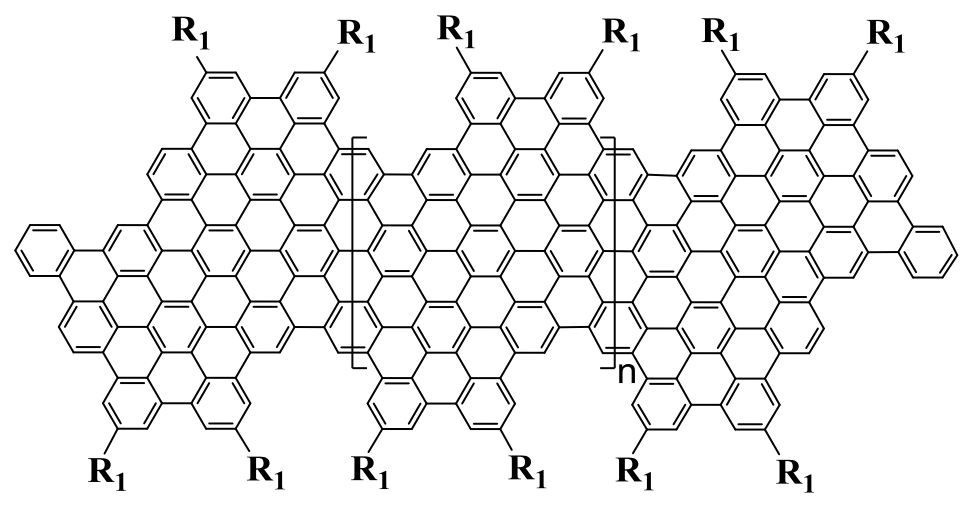

GNR-COOCH

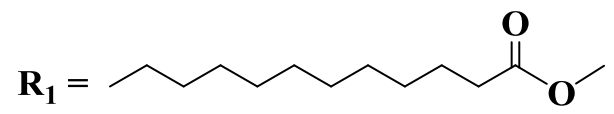

GNR-COOCH$_{3}$ samples (GNR-1, GNR-2 and GNR-3) were prepared via the similar cyclodehydrogenation method as mentioned above for the preparation of C78 in Section 4. Briefly, $\mathrm{PP}-\mathrm{COOCH}_{3}(50.0 \mathrm{mg})$ was dissolved in $50.0 \mathrm{ml}$ of dichloromethane. Followed by the addition of ferric chloride $(920 \mathrm{mg}, 5.7 \mathrm{mmol}, 7.5$ eqv./H) dissolved in $2.0 \mathrm{ml}$ of nitromethane. Then a stream of nitrogen saturated with dichloromethane was passed through the reaction mixture for $2 \mathrm{~h}$. The mixture was stirred at room temperature for $24 \mathrm{~h}$. Finally, an excess amount of methanol was added, which resulted in the formation of precipitates. The precipitates were collected by extraction filtration and washed with water and methanol for at least 3 circles. After vacuum drying, $47.0 \mathrm{mg}$ of a black solid was obtained in yield of $93 \%$. 


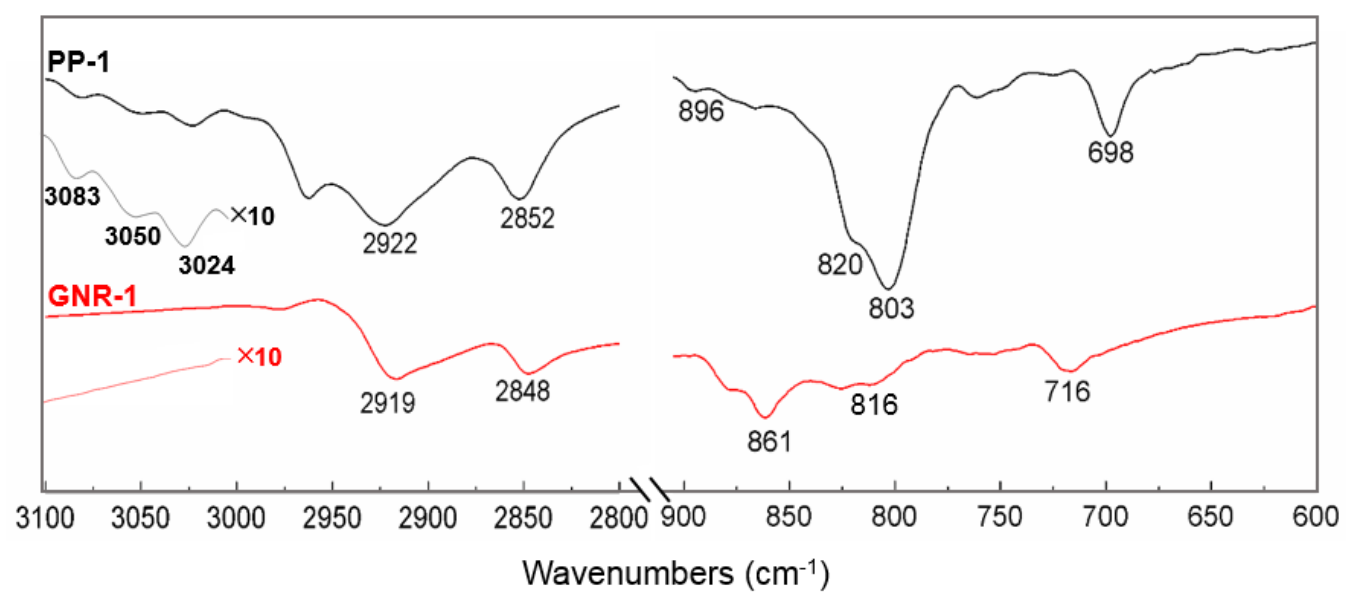

Figure S10. FTIR spectral regions for PP-1 and GNR-1. The great attenuation of the signals from aromatic $\mathrm{C}-\mathrm{H}$ stretching vibrations at 3083,3050 and $3024 \mathrm{~cm}^{-1}$ in GNR-1 spectrum, the disappearance of bands at 820,803 and $698 \mathrm{~cm}^{-1}$, which originate from mono- and disubstituted benzene rings, and the appearance of bands for aromatic $\mathrm{C}-\mathrm{H}$ at the edge positions at 861 and $816 \mathrm{~cm}^{-1}$ confirm the efficient cyclodehydrogenation of the polyphenylene precusor. ${ }^{3,4}$

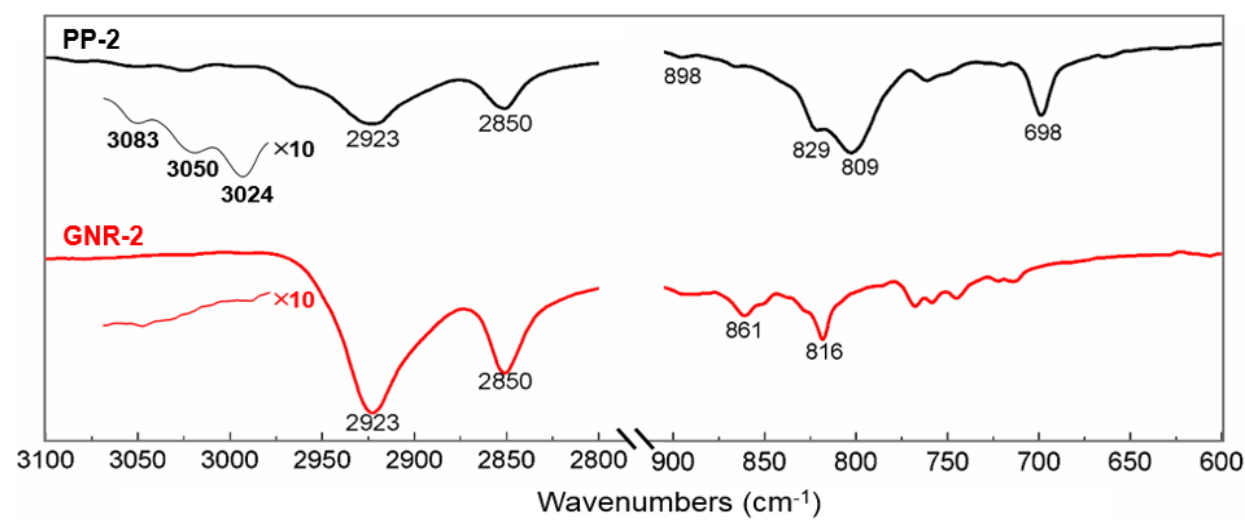

Figure S11. FTIR spectral regions for PP-2 and GNR-2. The evident attenuation of the peaks from aromatic $\mathrm{C}-\mathrm{H}$ stretching vibrations at 3083,3050 and $3024 \mathrm{~cm}^{-1}$ in spectrum of GNR-2, the disappearance of bands at 829,809 and $698 \mathrm{~cm}^{-1}$ that attributed to mono- and disubstituted benzene rings, and the appearance of bands for aromatic $\mathrm{C}-\mathrm{H}$ at the edge positions at 861 and $816 \mathrm{~cm}^{-1}$ confirm the cyclodehydrogenation of the polyphenylene precursor. ${ }^{3,4}$ 


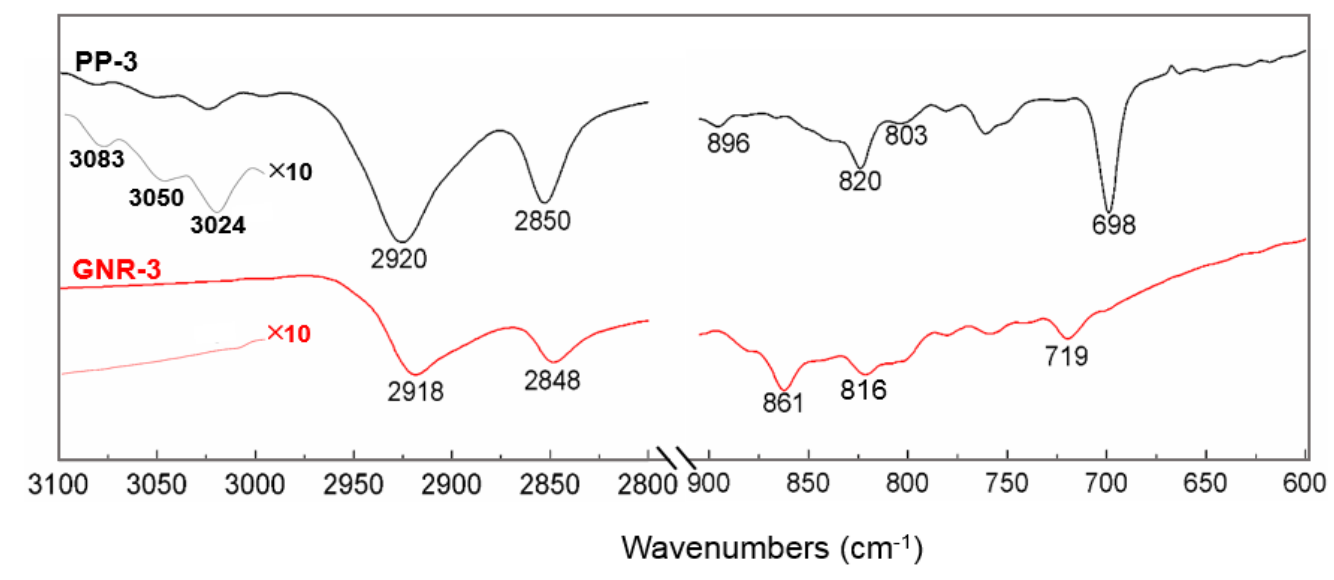

Figure S12. FTIR spectral regions for PP-3 and GNR-3. The evident attenuation of the peaks from aromatic $\mathrm{C}-\mathrm{H}$ stretching vibrations at 3083,3050 and $3024 \mathrm{~cm}^{-1}$ in spectrum of GNR-3, the disappearance of bands at 820,803 and $698 \mathrm{~cm}^{-1}$ that attributed to mono- and disubstituted benzene rings, and the appearance of bands for aromatic $\mathrm{C}-\mathrm{H}$ at the edge positions at 861 and $816 \mathrm{~cm}^{-1}$ confirm the cyclodehydrogenation of the polyphenylene precursor. ${ }^{3,4}$

\subsection{Synthesis of GNR-COOH}

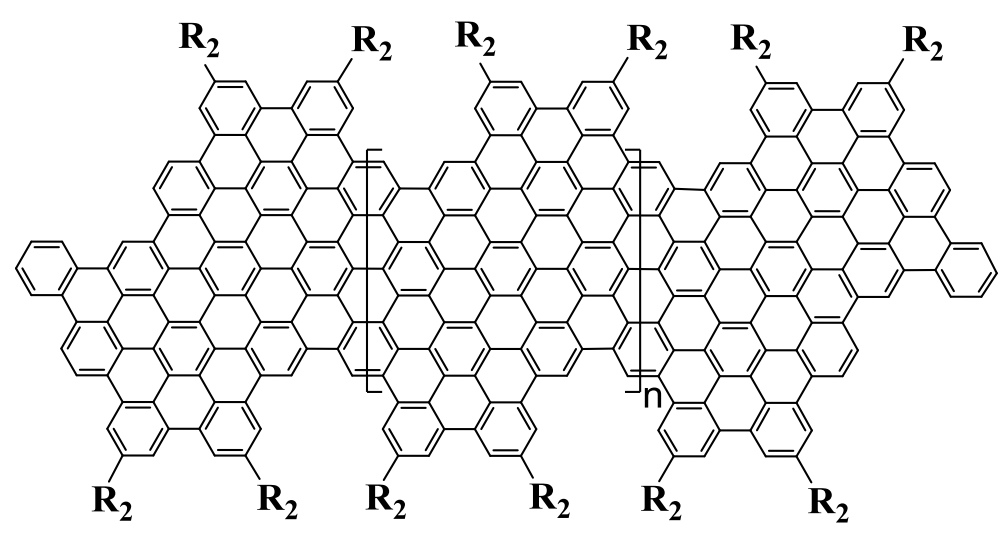

GNR-COOH<smiles>[R]C=CCCCCCCCCCCC(=O)O</smiles> 
GNR-COOCH $3($ GNR-2, $30 \mathrm{mg})$ and potassium hydroxide $(0.9 \mathrm{~g})$ were dissolved in a mixed solvent of $40 \mathrm{~mL}$ THF, $4 \mathrm{~mL}$ methanol and $4 \mathrm{~mL}$ water. Subsequently, the mixture was refluxed for $24 \mathrm{~h}$ and then cooled down to room temperature. Then the mixture was neutralized with $2 \mathrm{M} \mathrm{HCl}$ aqueous solution. The black precipitate was collected by extraction filtration and then washed with water, THF and methanol. After vacuum drying at room temperature, $26 \mathrm{mg}$ GNR-COOH was obtained ( $\sim 97 \%$ yield). The comparison of the FTIR spectra of GNR-COOCH 3 and GNR-COOH is given below in Figure S13, which demonstrates the successful conversion of $\mathrm{GNR}_{-} \mathrm{COOCH}_{3}$ to GNR-COOH.

\subsection{Synthesis of GNR-PEO ${ }^{2}$}

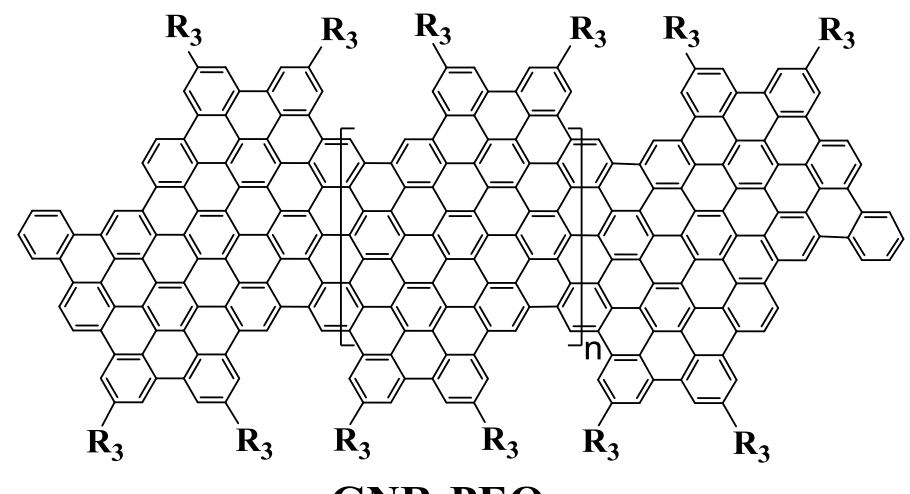

GNR-PEO

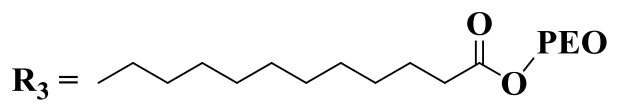

GNR-COOH (GNR-2I, $10 \mathrm{mg}, 6.0 \times 10^{-3} \mathrm{mmol}$ repeat units) and poly(ethylene oxide) (PEO, $\left.M_{\mathrm{n}} 1000,120 \mathrm{mg}, 0.12 \mathrm{mmol}\right)$ were placed into a dry $100 \mathrm{~mL}$ Schlenk tube, a mixed solvent of dry THF (12 mL) and DMF (6 mL) were added into the tube. Sonication was employed to obtain a homogeneous GNR-COOH dispersion, in which PEO was soluble. Then, dimethylaminopyridine (DMAP, $0.44 \mathrm{mg}, 3.6 \times 10^{-3} \mathrm{mmol}$ ) 
was added and the mixture was stirred for several minutes. After the mixture was cooled to $0{ }^{\circ} \mathrm{C}$, 1-(3-dimethylaminopropyl)-3-ethylcarbodiimide hydrochloride (EDC, $9.5 \mathrm{mg}, 5.0 \times 10^{-2} \mathrm{mmol}$ ) was added. The mixture was stirred at $0{ }^{\circ} \mathrm{C}$ for $30 \mathrm{~min}$, and finally at room temperature for 3 days. Afterwards, the mixture was dialyzed (use a dialysis membrane with cutoff of $100 \mathrm{kDa}$ ) against pure water by changing the external water for more than 10 times over a period of 3 days. Finally, black product was collected by extraction filtration and then dried under vacuum at room temperature for 24 hours.

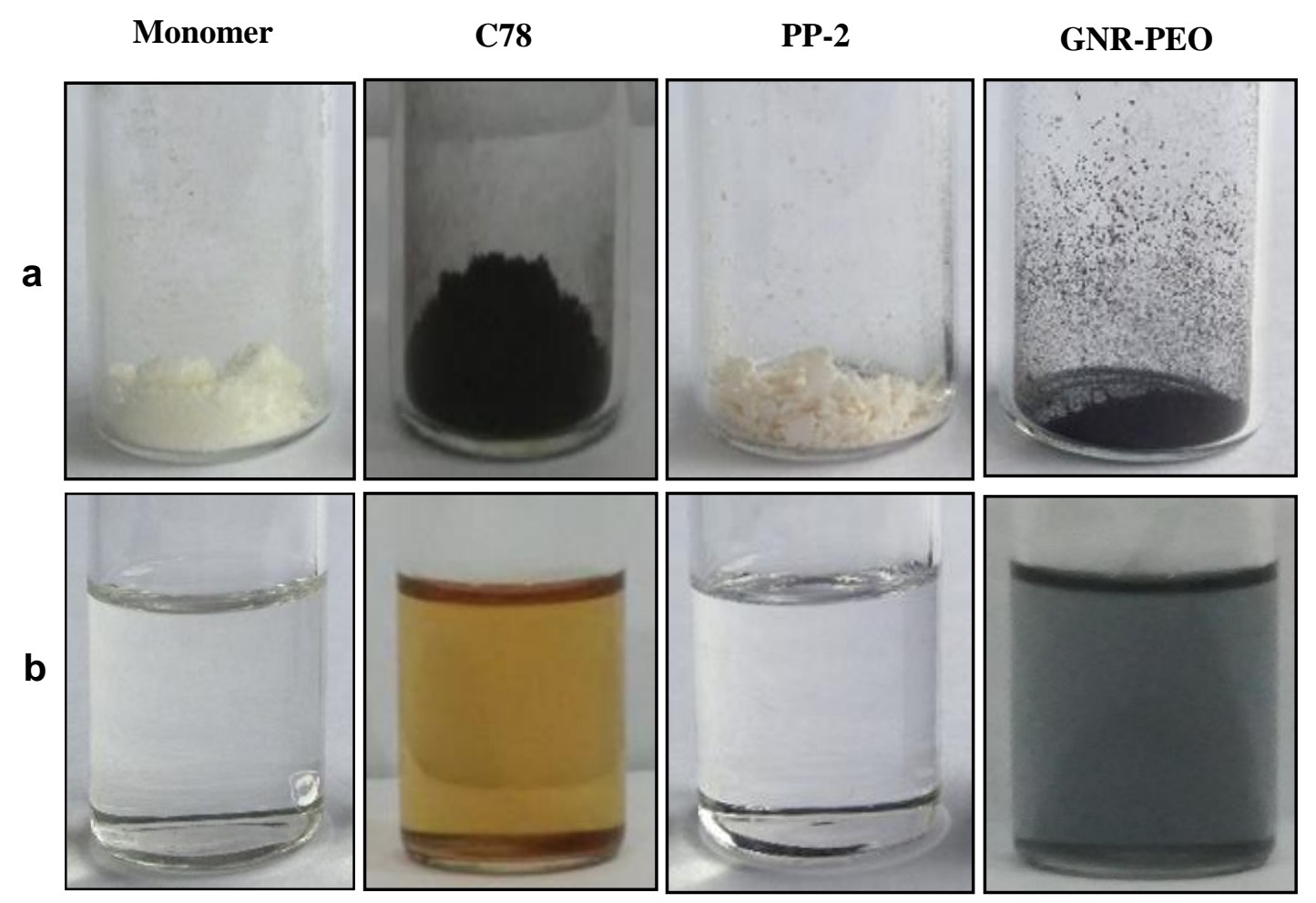

Figure S13. Photographs of the powder samples of the monomer, C78, PP-2 and GNR-PEO (a), as well as the corresponding THF solutions of the monomer, C78, PP-2 and the THF dispersion of GNR-PEO (b). 


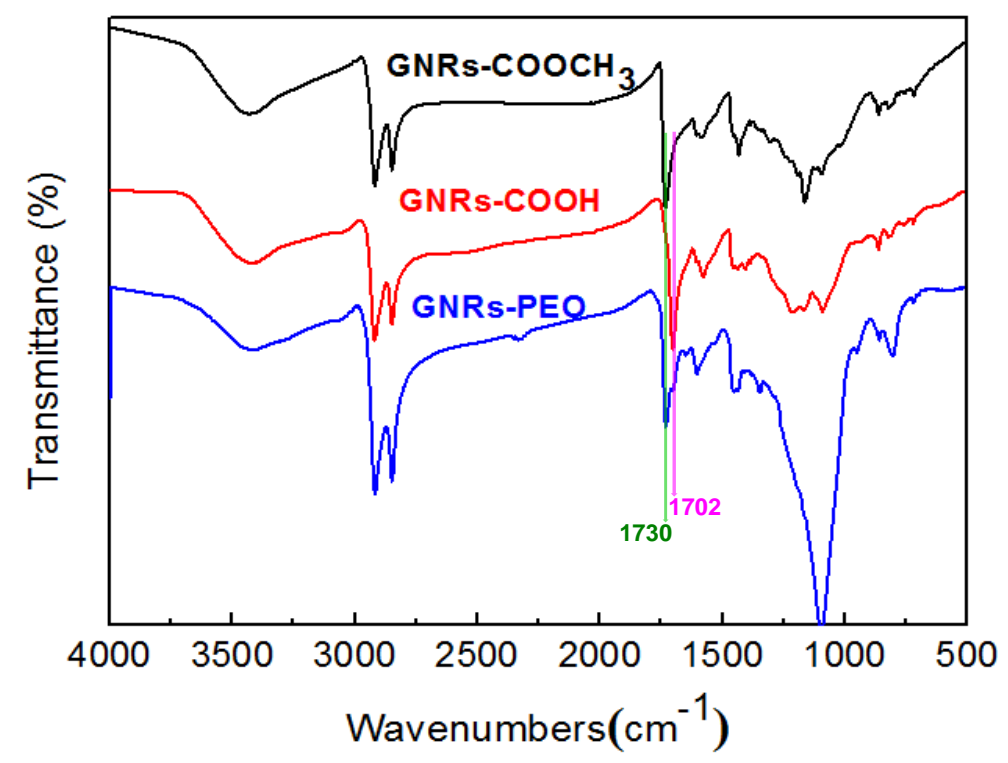

Figure S14. FTIR spectra of GNR-COOCH 3 , GNR-COOH and GNR-PEO. The increase of the intensity ratio of the absorption band at $1730 \mathrm{~cm}^{-1}$ (the stretching of $\mathrm{C}=\mathrm{O}$ in an eater group) to that at $1702 \mathrm{~cm}^{-1}$ (the stretching of $\mathrm{C}=\mathrm{O}$ in a carboxy group) demonstrates the successful grafting of the PEO chains.

\section{Solid-State NMR measurements of $\mathrm{PP}-\mathrm{COOCH}_{3}, \mathrm{GNR}^{-\mathrm{COOCH}_{3}}$ and GNR-PEO samples}

All solid-state ${ }^{1} \mathrm{H}$ magic-angle-spinning (MAS) experiments were performed on a Bruker AVANCE III 700 (16.4 T, $700 \mathrm{MHz}$ for ${ }^{1} \mathrm{H}$, Aarhus University) spectrometer using a $1.3 \mathrm{~mm}$ Bruker probe operating in double-resonance mode. The ${ }^{13} \mathrm{C}\left\{{ }^{1} \mathrm{H}\right\}$ cross-polarization $(\mathrm{CP})^{6}$ solid-state MAS experiment shown in Fig S15a was acquired on a Bruker AVANCE II $400\left(9.4 \mathrm{~T}, 400 \mathrm{MHz}\right.$ for ${ }^{1} \mathrm{H}$, Aarhus University) spectrometer using a $2.5 \mathrm{~mm}$ Bruker probe operating in double-resonance mode. The magic angle setting was done on $\mathrm{KBr}^{7}$ and referencing of resonances was done relative to tetramethylsilane (TMS), using adamantane as a secondary reference with ${ }^{13} \mathrm{C}_{\text {low-field }}$ at $38.48 \mathrm{ppm}$ and ${ }^{1} \mathrm{H}$ at $1.85 \mathrm{ppm} .{ }^{8,9}$ In the ${ }^{1} \mathrm{H}$ experiments, the MAS 
frequency was set to $59524 \mathrm{~Hz}$ giving a rotor period of $16.8 \mu \mathrm{s}$. The carrier on ${ }^{1} \mathrm{H}$ was set at $4 \mathrm{ppm}$ and all $\pi / 2$-pulses were set to last $1.8 \mu \mathrm{s}$, corresponding to a radio-frequency (rf) field of $139 \mathrm{kHz}$. For the samples $\mathrm{PP}-\mathrm{COOCH}_{3}, \mathrm{GNR}-\mathrm{COOCH}_{3}$ and GNR-PEO, the recycling delay was set to 3,1 , and $1.5 \mathrm{~s}$, respectively. In all experiments, the number of dummy scans extended over the entire phase cycle, which was (i) 4 steps in standard MAS NMR experiments and (ii) 16 steps in case of ${ }^{1} \mathrm{H}-{ }^{1} \mathrm{H}$ double quantum-single quantum (DQ-SQ) correlation experiments. All experiments were recorded at ambient conditions. To reduce the ${ }^{1} \mathrm{H}$ background of the probe in the displayed standard ${ }^{1} \mathrm{H}$ MAS NMR spectrum, the procedure proposed by Chen et al. ${ }^{10}$ was invoked. In the ${ }^{1} \mathrm{H}-{ }^{1} \mathrm{H}$ DQ-SQ MAS NMR correlation experiments, DQ recoupling and DQ reconversion were achieved using the Back-to-Back $(\mathrm{BaBa})$ recoupling sequence ${ }^{11,12}$ employing four rotor periods on $\mathrm{PP}-\mathrm{COOCH}_{3}$ and two rotor periods on the GNR samples. The dwell time in the indirect dimension was set to one rotor period. Processing was done in Topspin 3.2. Phase correction was done manually and window functions were imposed in the ${ }^{1} \mathrm{H}-{ }^{1} \mathrm{H}$ DQ-SQ correlation spectra. A Gaussian multiplication using a line broadening parameter of -20 was applied and the fraction parameter was set such that the windowing maximum was in the beginning of the active fid but after the initial maximum. In the ${ }^{13} \mathrm{C}\left\{{ }^{1} \mathrm{H}\right\} \mathrm{CP} / \mathrm{MAS}$ NMR experiment on GNR-COOCH 3 , the MAS frequency was set to $20.0 \mathrm{kHz}$ giving a rotor period of $50.0 \mu \mathrm{s}$. The carrier frequency on ${ }^{13} \mathrm{C}$ and ${ }^{1} \mathrm{H}$ was set at $100 \mathrm{ppm}$ and 4 ppm, respectively. All rf-field calibrations and the search for an optimal first-order Hartmann-Hahn match using a 70-100\%-RAMP on the ${ }^{1} \mathrm{H}$ channel were performed on 
uniformly ${ }^{13} \mathrm{C},{ }^{15} \mathrm{~N}$-labeled L-Alanine. The excitation pulse on the ${ }^{1} \mathrm{H}$ nuclei was set to $3.25 \mu \mathrm{s}$ and the nominal rf-field value of the ${ }^{1} \mathrm{H} \mathrm{CP}$ ramp was $88 \mathrm{kHz}$. A CP $3.0 \mathrm{~ms}$ mixing time was used to probe the solid/flexible ${ }^{13} \mathrm{C}$ nuclei with and without directly attached protons. The ${ }^{13} \mathrm{C}\left\{{ }^{1} \mathrm{H}\right\} \mathrm{CP} / \mathrm{MAS}$ NMR experiment employed an eight-step phase cycling and ${ }^{1} \mathrm{H}$ decoupling during acquisition was achieved using the UTPD $\left(170^{\circ}, 0.92 \mathrm{t}_{\mathrm{r}}, 1.03 \mathrm{t}_{\mathrm{r}}\right)^{13}$ scheme with an rf field of $88 \mathrm{kHz} .36000$ transients were co-added and the recycling delay was set to 2 s. The ${ }^{13} \mathrm{C}\left\{{ }^{1} \mathrm{H}\right\}$ CP/MAS NMR experiment was recorded under ambient conditions. Processing was done in Topspin 3.2. Phase correction was done manually, a window function with a Lorentzian broadening of $10 \mathrm{~Hz}$ was imposed and automatic baseline correction was used.

The quantitative Single-Pulse/MAS (SP/MAS) solid-state ${ }^{13} \mathrm{C}$ NMR experiment of GNR-PEO was performed on a Bruker AVANCE III 300 WB spectrometer (Shanghai Key Laboratory of Magnetic Resonance, East China Normal University) operating at 75.5 $\mathrm{MHz}$ for ${ }^{13} \mathrm{C}$. A $4 \mathrm{~mm}$ double resonance MAS probe was used for the experiment. The MAS frequency was set to $10.0 \mathrm{kHz}$ giving a rotor period of $100.0 \mu \mathrm{s}$. The carrier frequency on ${ }^{13} \mathrm{C}$ and ${ }^{1} \mathrm{H}$ was set at $6808.3 \mathrm{~Hz}(\sim 90 \mathrm{ppm})$ and $6930.9 \mathrm{~Hz}(\sim 8 \mathrm{ppm})$, respectively. The excitation pulse on the ${ }^{13} \mathrm{C}$ nuclei was set to $4.0 \mu$ s corresponding to a flip angle of $90^{\circ} .1024$ transients were co-added and the recycle delay was set to 100 $\mathrm{S}$ to insure complete relaxation of the ${ }^{13} \mathrm{C}$ spins. High power ${ }^{1} \mathrm{H}$ decoupling (tppm15) ${ }^{14}$ of $62.5 \mathrm{kHz}$ was used during acquisition. The ${ }^{13} \mathrm{C}$ chemical shifts were calibrated using adamantane $\left(\delta_{\text {iso }}=38.56 \mathrm{ppm}\right)$. The experiment was recorded at room temperature. Processing was done in Topspin 3.1. Phase correction was done 
manually, a window function with a Lorentzian broadening of $10 \mathrm{~Hz}$ was imposed and automatic baseline correction was used. The integrals were evaluated using the build-in integrate function in Topspin.

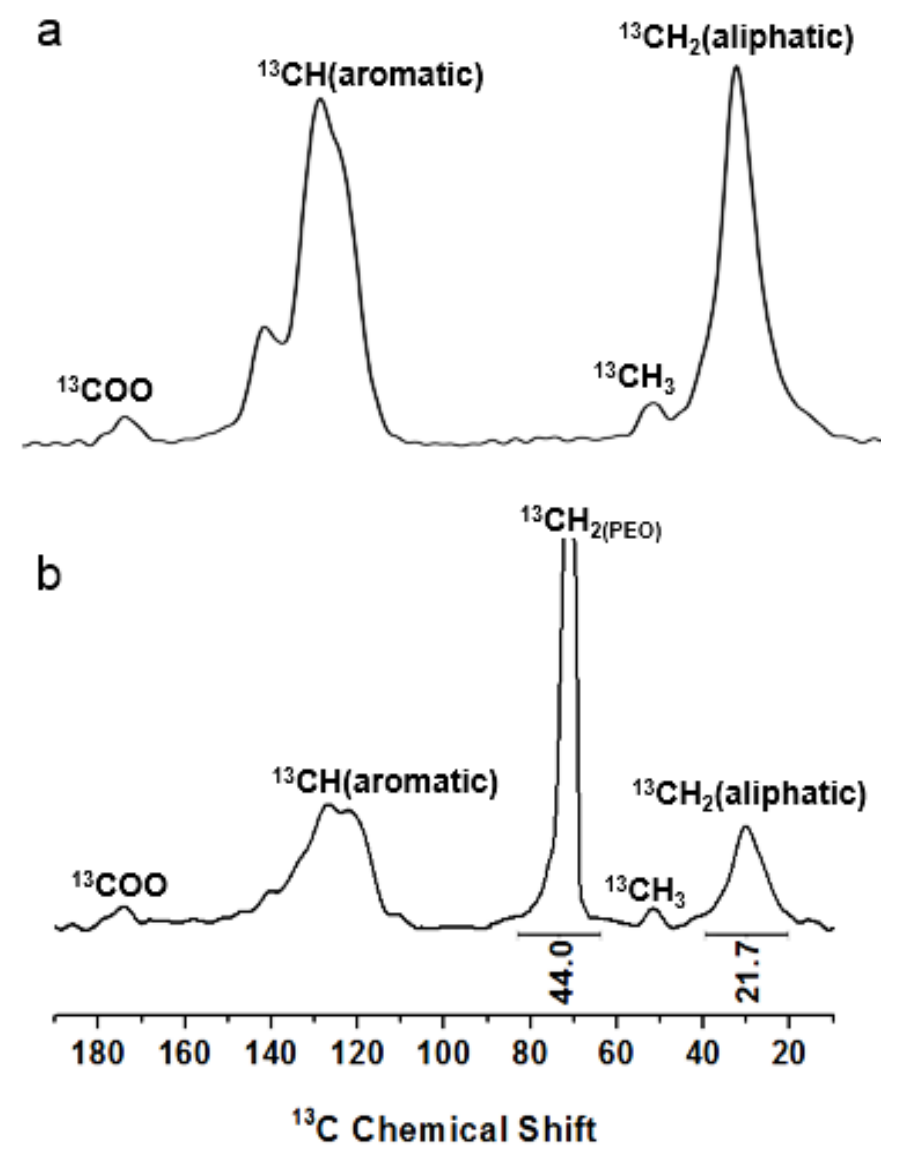

Figure S15. (a) The ${ }^{13} \mathrm{C}\left\{{ }^{1} \mathrm{H}\right\} \mathrm{CP} / \mathrm{MAS}$ NMR spectrum of GNR-COOCH${ }_{3}$ with $\mathrm{CP}$ times of $3.0 \mathrm{~ms}$ was acquired at $9.4 \mathrm{~T}$ using a MAS frequency of $20.0 \mathrm{kHz}$. (b) The ${ }^{13} \mathrm{C}$ SP/MAS NMR spectrum of GNR-PEO was acquired at $75.5 \mathrm{MHz}$ using a MAS frequency of $10.0 \mathrm{kHz}$. The ${ }^{13} \mathrm{CH}_{2 \text { (PEO) }}$ signal is cutoff at approximately $75 \%$ of the full intensity.

Calculation of the graft percentage (GP) of GNR-PEO:

The GP of GNR-PEO can be calculated from solid ${ }^{13} \mathrm{C}$ SP/MAS NMR (Figure S15b) via the Equation (1):

$$
\mathrm{GP}=\frac{I_{a}}{44} \times \frac{10}{I_{b}} \times 100 \%
$$


Where $I_{a}$ is the integral area of the C peak of PEO chains marked in the spectrum, $I_{b}$ represents the integral area of the $\mathrm{C}$ peak of the aliphatic groups labeled in the spectrum. According to the Equation (1), the GP of GNR-PEO is calculated to be $46 \%$.

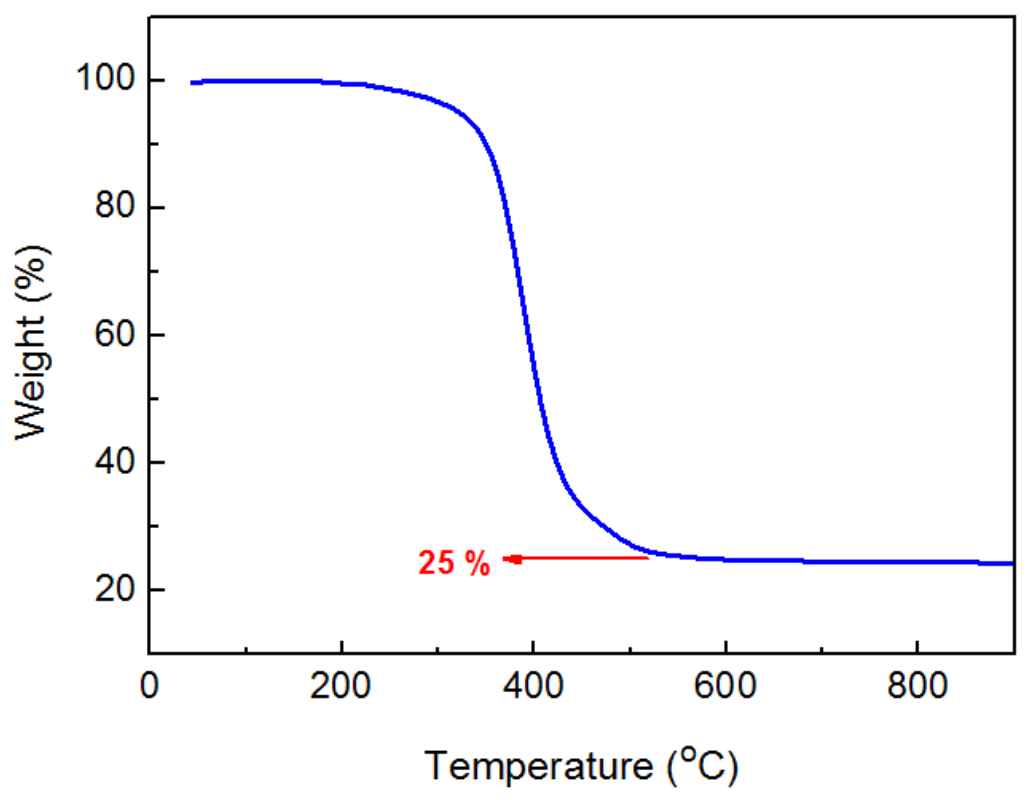

Figure S16. TGA curve of GNR-PEO

The GP of GNR-PEO can also be estimated according to the result of TGA (Figure S16).The corresponding Equation for GP is:

$$
\begin{aligned}
& W_{\text {PEO }}=1-W_{\text {residue }} \times \frac{186 \times 4}{948}-W_{\text {residue }} \\
& \mathrm{GP}=\frac{\frac{W_{P E O}}{1000}}{\frac{W_{\text {residue }}}{948} \times 4}
\end{aligned}
$$

where $W_{\text {residue }}$ is the weight fraction of the GNR rigid backbone, which still retained after thermal treatment over $500{ }^{\circ} \mathrm{C}$ according to the TGA curve; 948, 186 and 1000 represent the molar mass of GNR backbone, $\left(\mathrm{CH}_{2}\right)_{10} \mathrm{COO}$ and $\mathrm{PEO}$, respectively; $W_{\text {PEO }}$ denotes the weight fraction of the PEO chains. The GP of GNR-PEO, based on the Equation (3), is calculated to be $\sim 50 \%$. 
a

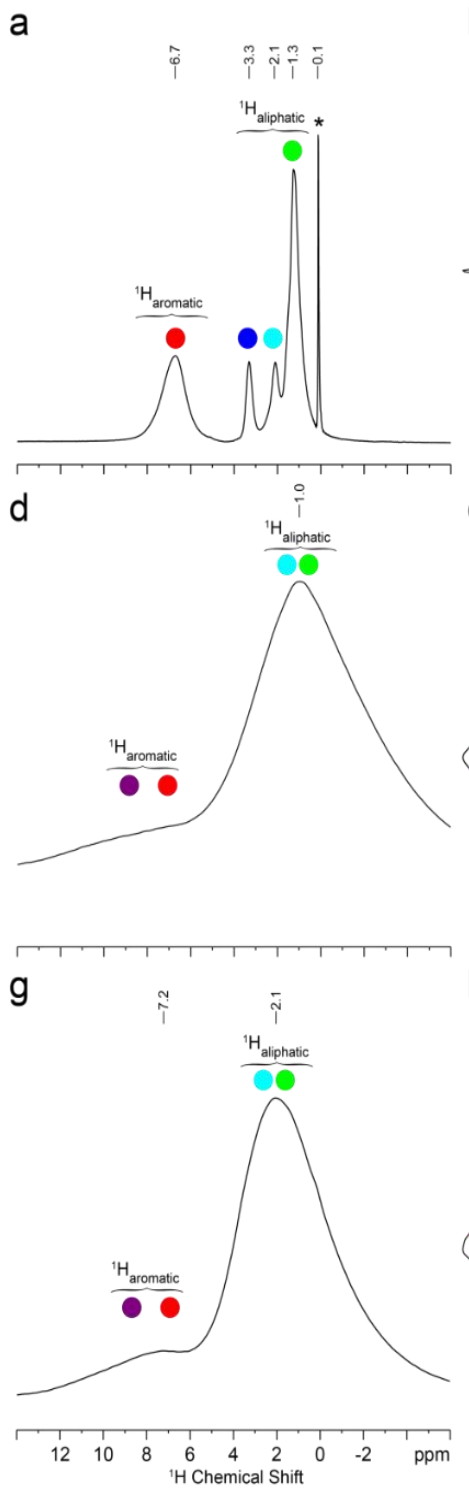

b
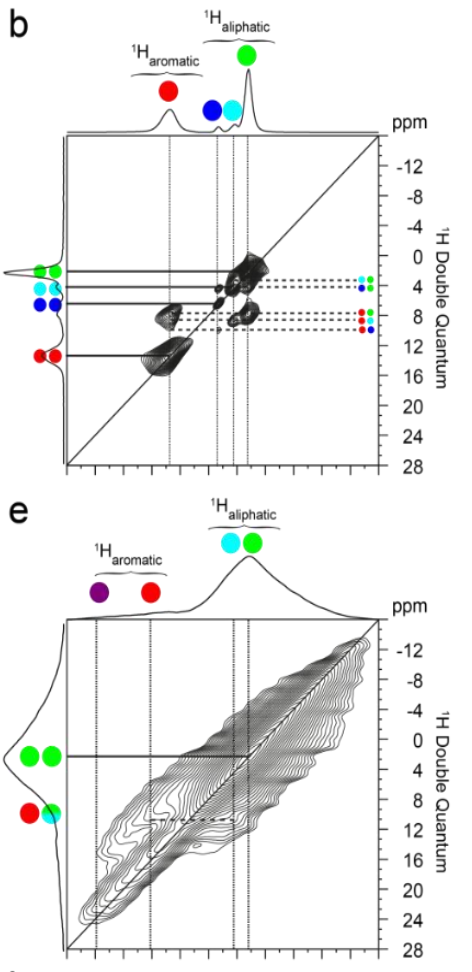

$\mathrm{h}$

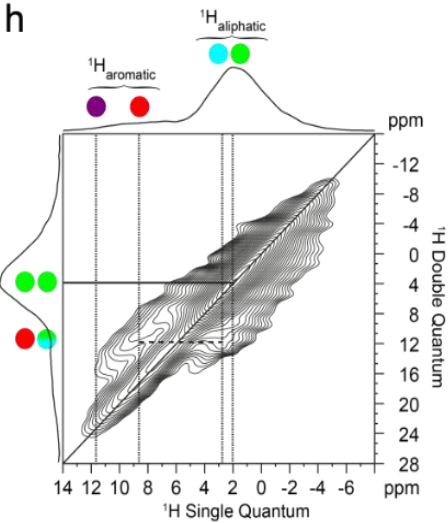

C

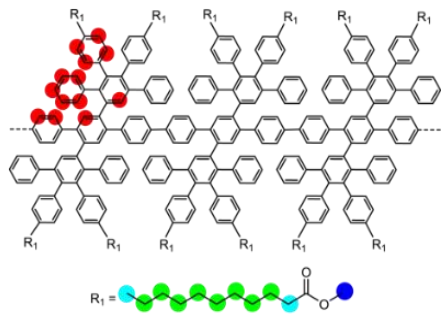

f

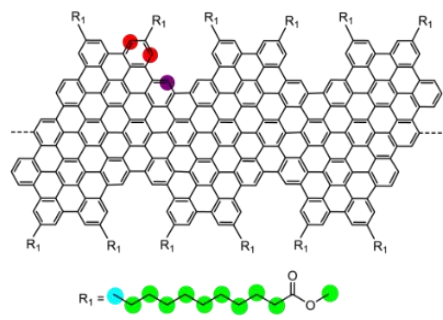

i

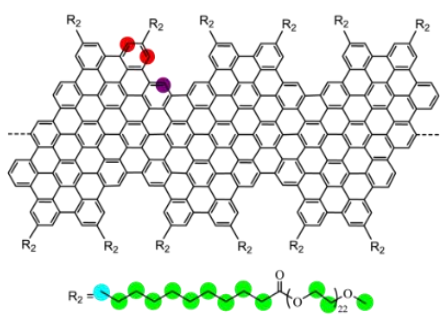

Figure S17. (a, d and g) Standard ${ }^{1} \mathrm{H}$ MAS NMR spectra with reduced ${ }^{1} \mathrm{H}$ background of the probe and rotor, (b, e and h) 2D ${ }^{1} \mathrm{H}-{ }^{1} \mathrm{H}$ DQ-SQ MAS NMR correlation spectra with (b) four rotor periods and (e and h) two rotor periods of back-to-back DQ recoupling and (c, f and i) assignment schemes of (a, b and c) $\mathrm{PP}^{-\mathrm{COOCH}_{3}}$, (d, e and f) GNR-COOCH${ }_{3}$ and ( $\mathrm{g}$, h and i) GNR-PEO. The asterisk in (a) signifies an impurity. The contour levels in (b) are shown from $1.5 \%$ to $87 \%$ and the contour levels in (e) and (h) are shown from $3 \%$ to $99 \%$ with respect to the internal maximum intensity. All spectra were recorded at $16.4 \mathrm{~T}$ using a MAS frequency of $59524 \mathrm{~Hz}$.

Solid-state ${ }^{1} \mathrm{H}$ MAS NMR spectroscopy allows for further characterizations of PP-COOCH 3 , GNR-COOCH 3 and GNR-PEO (Figure S17). PP-COOCH 3 exhibits a narrow signal at $\sim 6.7 \mathrm{ppm}$ from aromatic protons, suggesting a flexible structure. ${ }^{12-14}$ 
In sharp contrast, GNR-COOCH 3 and GNR-PEO show significantly broadened and shifted aromatic and aliphatic ${ }^{1} \mathrm{H}$ resonances, which verify the cyclodehydrogenation of the polyphenylene, yielding rigid and planar GNRs with extended $\pi$-conjugation. ${ }^{15-17,18}$ In addition, the aliphatic proton signals centered at $\sim 2.1 \mathrm{ppm}$ in GNR-PEO shift to higher ppm values compared to that of $\mathrm{GNR}-\mathrm{COOCH}_{3}$, which is ascribed to the $\mathrm{CH}_{2}$ protons in the PEO chains (Figure S17d, g). Furthermore, 2D ${ }^{1} \mathrm{H}-{ }^{1} \mathrm{H}$ double-quantum-single-quantum (DQ-SQ) correlation experiments were also performed to detect the spatial proximity between different protons via dipole-dipole interactions. In agreement with the relatively flexible structure, the $2 \mathrm{D}{ }^{1} \mathrm{H}-{ }^{1} \mathrm{H}$ DQ-SQ correlation spectrum of $\mathrm{PP}-\mathrm{COOCH}_{3}$ shows narrow ${ }^{1} \mathrm{H}-{ }^{1} \mathrm{H}$ signals between the aromatic protons as well as between the aromatic and aliphatic protons (Figure S17b). ${ }^{19,20}$ In comparison, due to the planarization and graphitization of the polyphenylene, the GNRs exhibits broad, stretched and split ridge correlation signals in the aromatic region, which are ascribed to the interactions between the only two types of aromatic protons that generate the higher and lower frequency parts of the aromatic signals, respectively (Figure S17e). In the 2D spectrum of GNR-PEO (Figure S17h), the tail does not extend as far down as in $\mathrm{GNR}-\mathrm{COOCH}_{3}$, which is attributed to the shift in resonance positions induced by the PEO chains.

\section{Raman spectroscopy analysis}

Raman spectroscopy was performed in backscattering geometry with an XploRA PLUS Confocal Raman Microscope by Horiba, equipped with several laser lines. 
Here we used $532 \mathrm{~nm}$ and $638 \mathrm{~nm}$ laser lines. The laser power was kept well below $0.1 \mathrm{~mW}$ to avoid heating or damaging the sample. The laser spot was focused on the grain powder sample with a $100 \times$ long-distance objective. Note that the RLBM was observed at both $532 \mathrm{~nm}$ and $638 \mathrm{~nm}$. However, the RLBM peak is well defined and intense only at $638 \mathrm{~nm}$, presumably because of resonance effects.

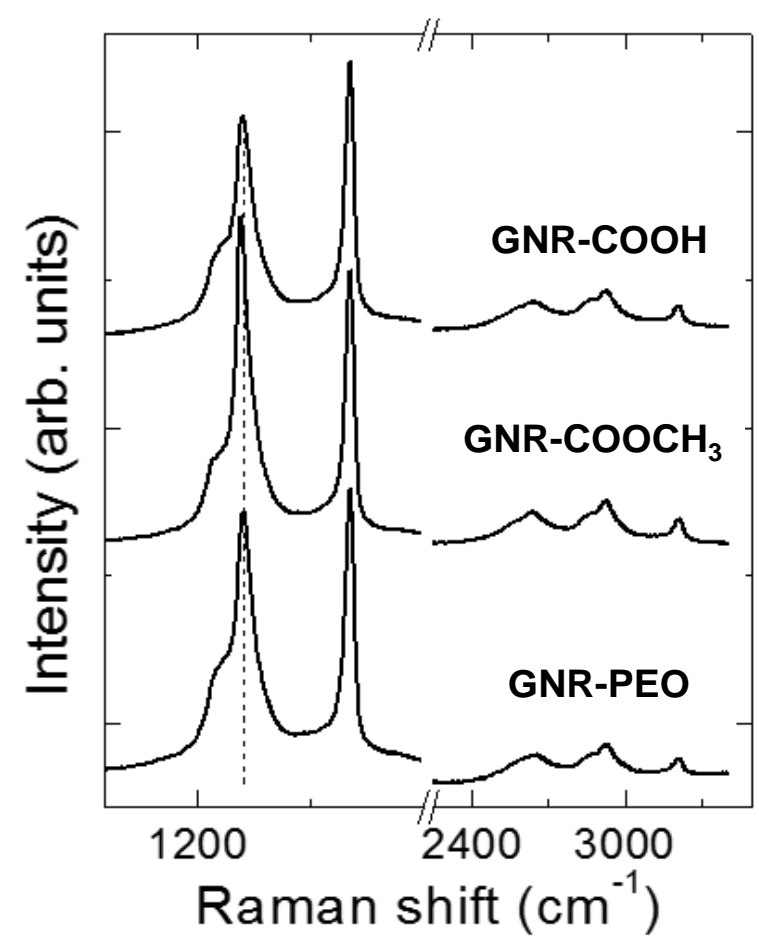

Figure S18. Raman spectra of GNRs upon excitation at $532 \mathrm{~nm}(2.33 \mathrm{eV})$ on a powder sample with laser power of $0.1 \mathrm{~mW}$. 


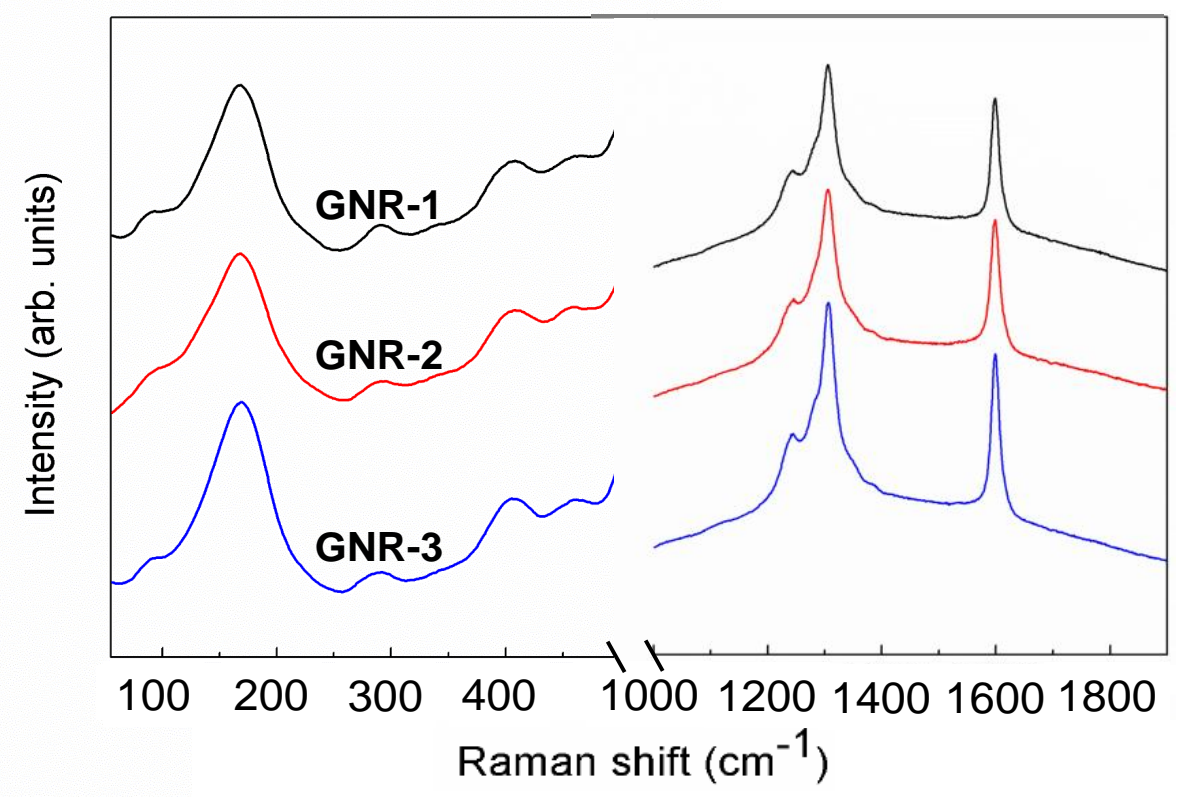

Figure S19. Raman (measured at $638 \mathrm{~nm}$ ) spectra of CNR-1, GNR-2 and GNR-3. This result suggests that the Raman spectrum is independent of the length of these GNRs.

8. Dispersibility of the GNRs in common organic solvents

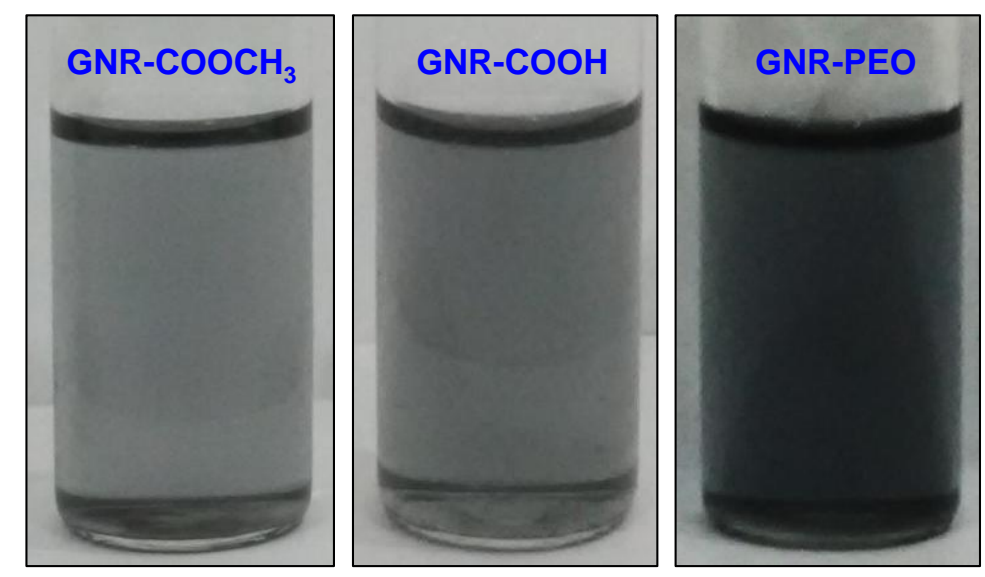

Figure S20. Photographs of GNR-COOCH 3 , GNR-COOH and GNR-PEO dispersions at the corresponding maximum concentrations in THF, respectively. 


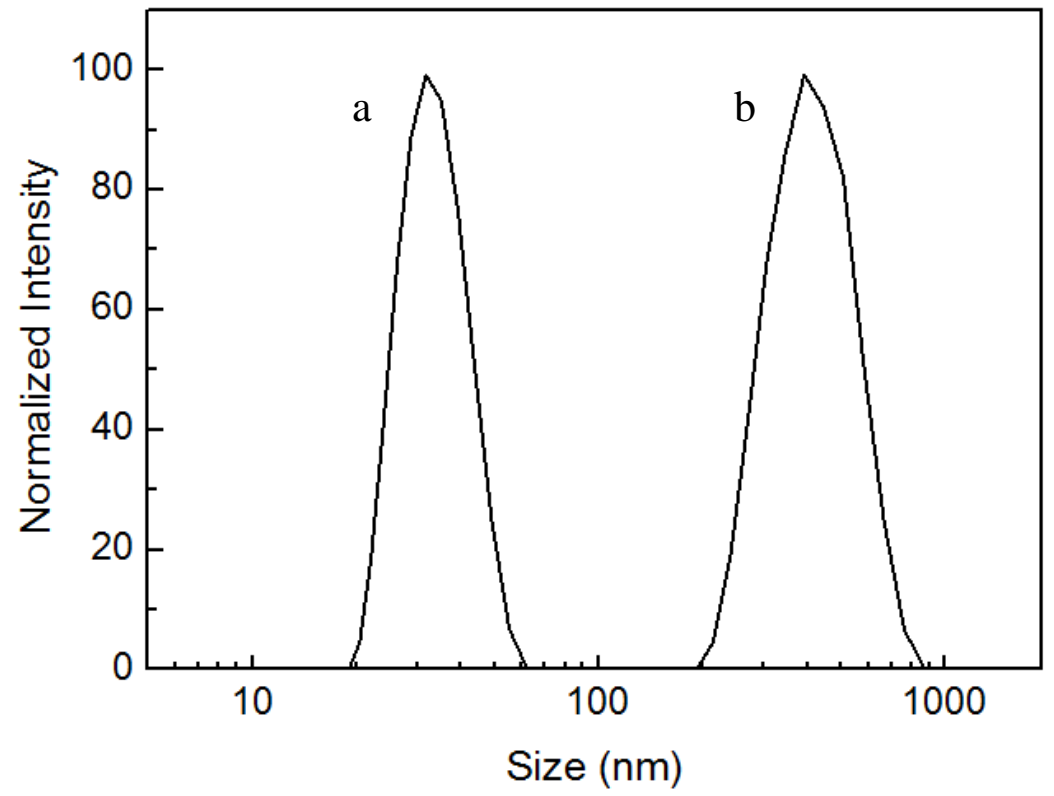

Figure S21. DLS plots of GNR-PEO dispersions in THF (a) and in $\mathrm{H}_{2} \mathrm{O}$ (b).

\section{Supramolecular nanostructures of GNR-PEO in $\mathrm{H}_{2} \mathrm{O}$}

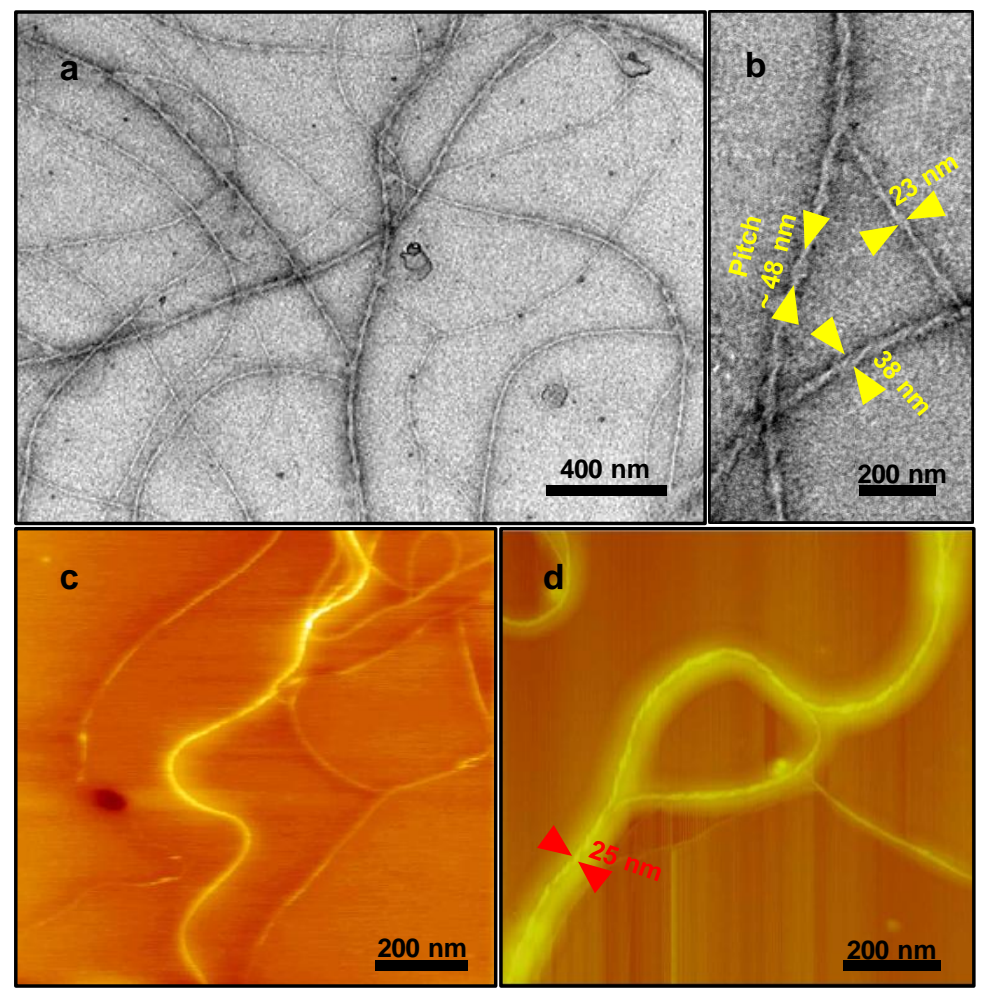

Figure S22. TEM (a, b) and AFM (c, d) images of supramolecular helical nanowires formed by the self-assembly of GNR-PEO in water. 
10. UV-Vis and fluorescence spectroscopy analyses

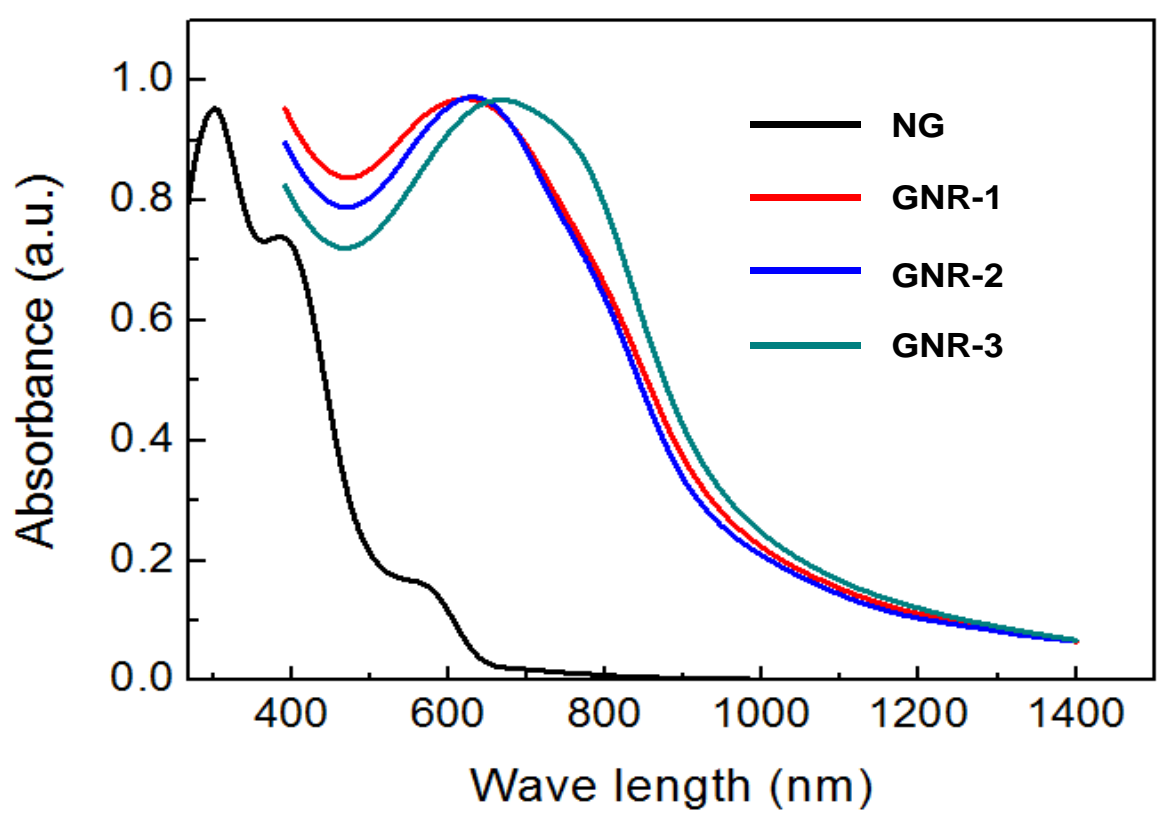

Figure S23. The UV-vis spectra of C78, GNR-1, GNR-2 and GNR-3 in THF (concentration: $0.01 \mathrm{mg} \mathrm{ml}^{-1}$ ).

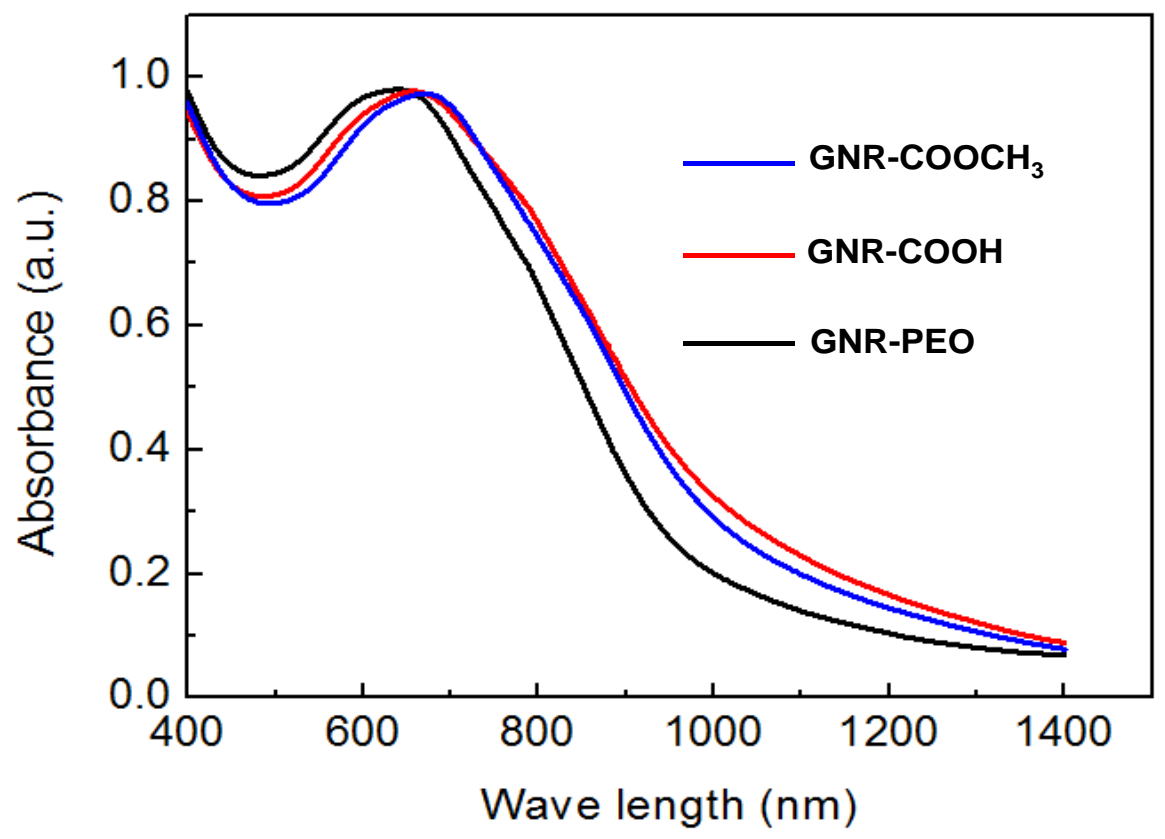

Figure S24. The UV-Vis spectra of GNR-COOCH 3 , GNR-COOH and GNR-PEO in NMP (concentration: $0.01 \mathrm{mg} \mathrm{ml}^{-1}$ ). 


\section{SPM visualization of the GNRs}

STM experiments carried out at the liquid-solid interface were performed using PicoLE (Agilent) instrument operating in constant-current mode with the STM tip immersed in the supernatant solution. HOPG (grade ZYB, Momentive Performance Material Quartz Inc., Strongsville, $\mathrm{OH}$ ) was used as substrate for STM as well as AFM measurements under ambient conditions. STM tips were prepared by mechanical cutting from Pt/Ir wire $(80 \% / 20 \%$, diameter $0.2 \mathrm{~mm})$. TCB was obtained from Sigma-Aldrich (99\%) and used without further purification. GNRs solutions were prepared by dispersing the GNR powder in TCB by ultrasonication for 24 hours and subsequent dilution of the mother solution; a droplet of the sample solution was applied by a glass pipette onto a freshly cleaved surface of HOPG. The imaging parameters are indicated in figure captions: sample bias $\left(V_{\text {bias }}\right)$ and tunneling current $\left(I_{\text {set }}\right)$.

AFM measurements were carried out using a Multimode AFM with a Nanoscope VIII controller (Veeco/Digital Instruments) or a Cypher AFM (Asylum Research) in intermittent contact mode. For AFM measurements, the diluted TCB solutions of GNRs were sonicated for $1 \mathrm{~h}$ and heated at $120{ }^{\circ} \mathrm{C}$ for $15 \mathrm{~min}$ prior to deposition. A drop of this hot solution was applied to a hot HOPG surface, which was also held at $120{ }^{\circ} \mathrm{C}$. After the hot deposition, the HOPG sample was heated for another $15 \mathrm{~min}$. The surface was then washed with $1 \mathrm{~mL}$ of TCB and heated again for $15 \mathrm{~min}$ to ensure complete evaporation of the solvent. The periodicity of the lamellae was measured both manually on the line profiles across the GNR domains and using the 
Fourier Transforms of the AFM images. Molecular models were constructed using the HyperChem 7.0 program. $^{21}$ STM and AFM images were processed using Scanning Probe Image Processor (SPIP, Image Metrology ApS) and WSxM (Nanotec Electronica, Spain) softwares, respectively.

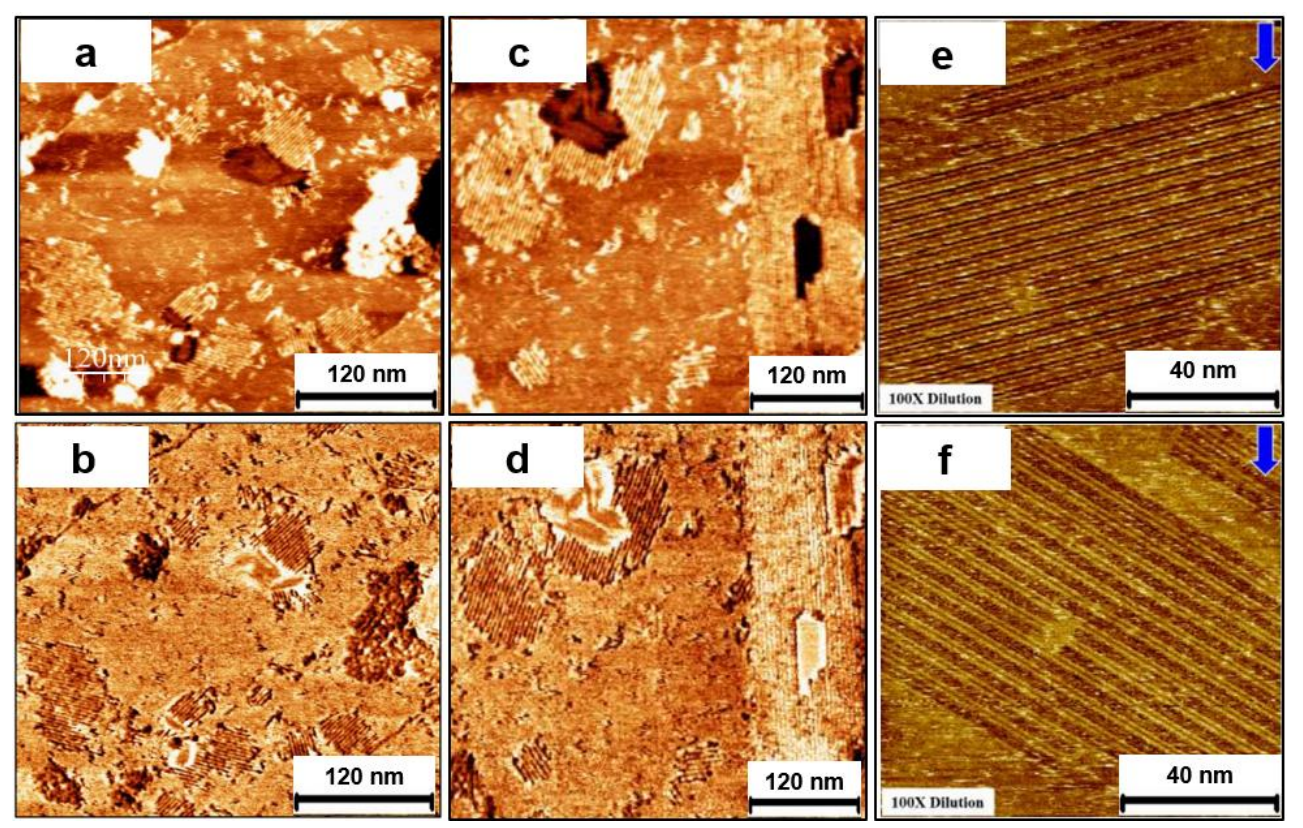

Figure S25. SPM analysis of self-assembled monolayers of GNR-PEO. (a, c) AFM topography images of isolated domains of GNR-PEO obtained on a dry film on HOPG. (b, d) AFM phase images of isolated domains of GNR-PEO from a dry film on HOPG. (e, f) STM image of isolated GNR-PEO domains at the TCB/HOPG interface (imaging parameters: $I_{\text {set }}=100 \mathrm{pA}, V_{\text {bias }}=-0.6 \mathrm{~V}$ ).

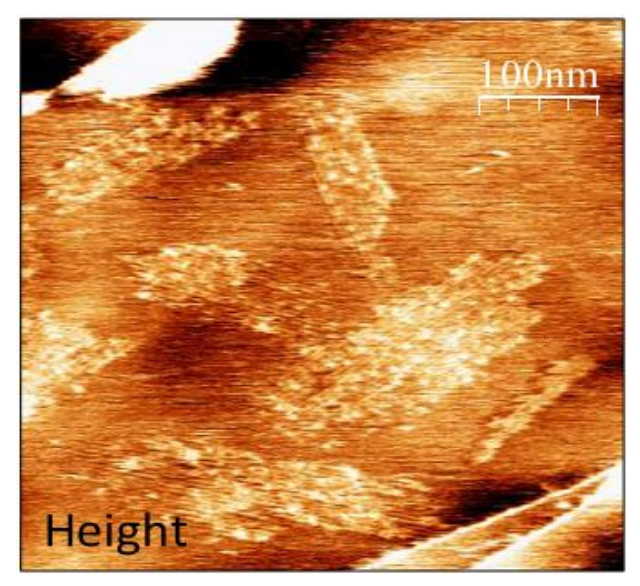

Figure S26. A typical AFM height image of isolated domain of GNR-COOH obtained on a dry film on HOPG (corresponding to the phase image in Fig. 2e in the main text). 


\section{GNR thin-film device fabrication and measurements}

$1 \mathrm{~nm} \mathrm{Ti} / 50 \mathrm{~nm} \mathrm{Au}$ electrodes were patterned on the top of $\mathrm{P}^{++} \mathrm{Si} / 300 \mathrm{~nm} \mathrm{SiO} 2$ substrate by employing E-beam lithography, metal deposition, and lifting-off techniques. Then the substrate was annealed in argon at $200{ }^{\circ} \mathrm{C}$ for $1 \mathrm{~h}$ to improve their contacts. Then, the THF dispersion of GNR-PEO, prepared by sonication for $1 \mathrm{~h}$, was dropped on the substrate, followed by heating for the solvent evaporation. Afterward, the sample was loaded into a furnace with $\mathrm{H}_{2}$-Ar gas mixture $(\mathrm{V}: \mathrm{V}=5: 95)$ at a low rate of $270 \mathrm{sccm}$, followed by heating to $500{ }^{\circ} \mathrm{C}$ and then annealing for $2 \mathrm{~h}$ under a pressure of 6 Torr. Electrical properties were tested on an Agilent semiconductor parameter analyzer (4156C) under high vacuum.
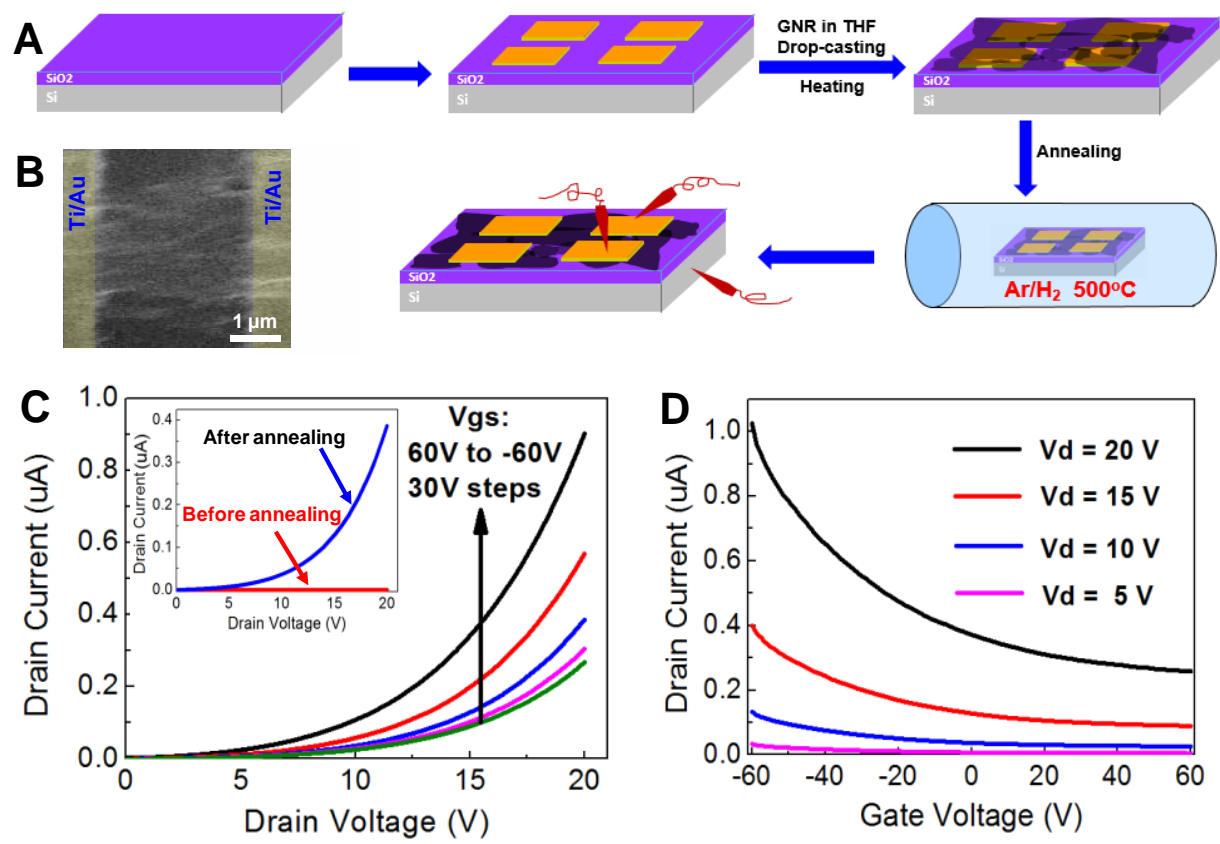

Figure S27. (A) Schematic illustration of the fabrication of a GNR thin-film based FET. (B) SEM image of the thin film between two Ti/Au electrodes (2nm-Ti/30nm-Au prepared by E-beam lithography and E-beam evaporation). (C) Current vs drain voltage $\left(I-V_{\mathrm{d}}\right)$ of the FET under different gate voltages $\left(V_{\mathrm{gs}}\right)$. Inset: $I-V_{\mathrm{d}}$ of the FET before (red) and after (blue) annealing at $500{ }^{\circ} \mathrm{C}\left(V_{\mathrm{gs}}=0 \mathrm{~V}\right)$. (D) $I-V_{\mathrm{gs}}$ of the FET under different drain voltages. 


\section{Calculation of the carrier mobility of the GNR thin film:}

The carrier mobility of the GNR thin film was calculated according to the following equation:

$$
\mu=\frac{L}{W C_{i} V_{S D}} \frac{\partial I_{S D}}{\partial V_{G}}
$$

Where $L$ is the length and $W$ is the width of the channel, $I_{S D}$ is the drain current, $V_{S D}$ is the drain voltage, $V_{G}$ is the so-called threshold gate voltage, and $C_{i}\left(1.1 \times 10^{-8} \mathrm{~F} / \mathrm{cm}^{2}\right)$ is the capacitance per unit area of the $300 \mathrm{~nm}$-thick $\mathrm{SiO}_{2}$ insulator layer.

A
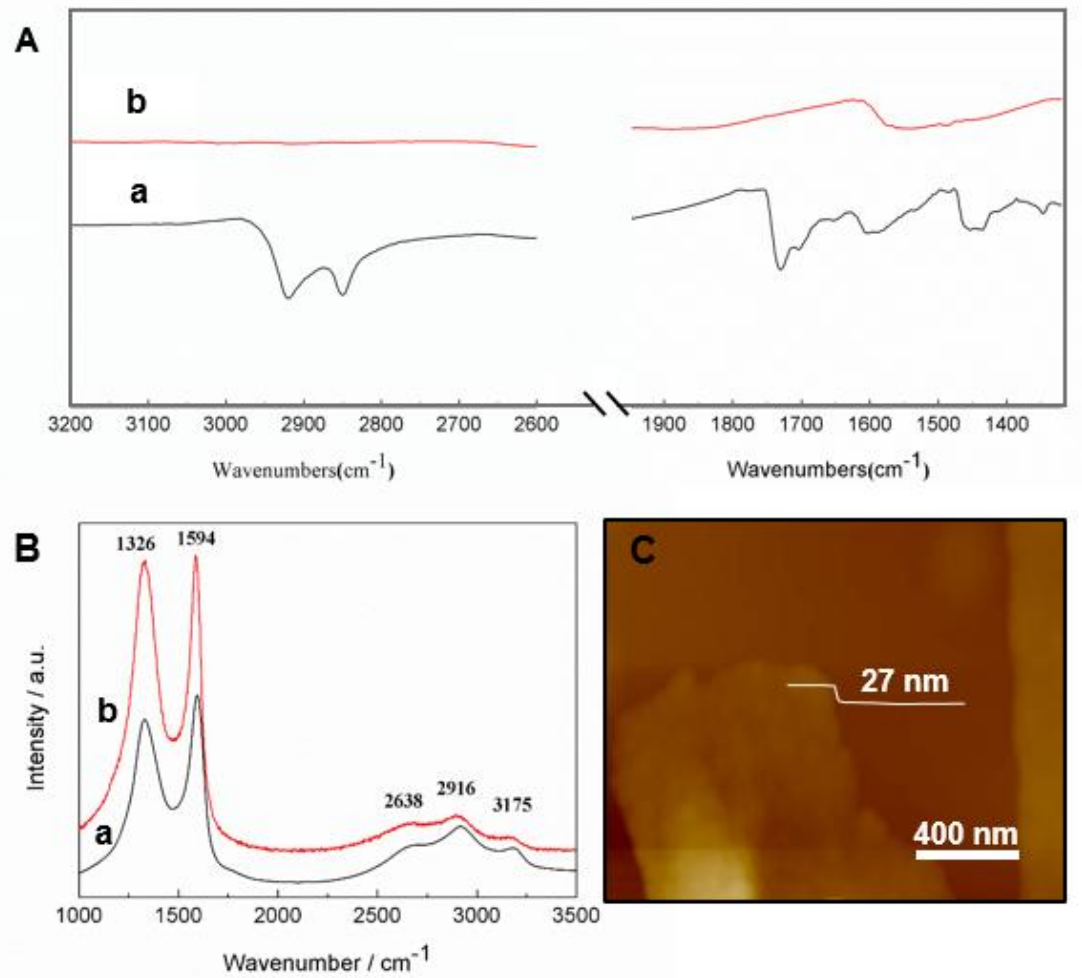

Figure S28. (A) ATR-FTIR spectra of pristine GNR-PEO (a) and annealed GNR films at $500{ }^{\circ} \mathrm{C}$ (b), the disappearance of the alkyl $\mathrm{C}-\mathrm{H}$ peaks (i.e. $\sim 1350,1450$, and $\left.2850-2925 \mathrm{~cm}^{-1}\right)$ and the $\mathrm{C}=\mathrm{O}$ peaks $\left(1732,1700 \mathrm{~cm}^{-1}\right)$ after annealing at $500^{\circ} \mathrm{C}$ suggests the removal of alkyl and PEO chains from the GNR edges at such conditions. (B) Raman spectra of pristine GNR-PEO (a) and annealed films at $500^{\circ} \mathrm{C}$ (b) measured at $532 \mathrm{~nm}(2.33 \mathrm{eV})$ with laser power below $0.1 \mathrm{Mw}$, showing D, G, 2D, and D+D' bands, in agreement with the GNR-COOCH 3 samples, which indicate that the annealing condition we used did not affect the GNR basal plane. (C) AFM height profile of the device showing a thickness of $\sim 27 \mathrm{~nm}$ of the GNR film. 


\section{References}

1. Schwab, M. G.; Narita, A.; Hernandez, Y.; Balandina, T.; Mali, K. S.; Feyter, S. D.; Feng, X.; Müllen, K. J. Am. Chem. Soc. 2012, 134, 18169.

2. Huang, Y.; Mai, Y.; Yang, X.; Beser, U.; Liu, J.; Zhang, F.; Yan, D.; Müllen, K.; Feng, X. J. Am. Chem. Soc. 2015, 137, 11602.

3. Shifrina, Z. B.; Averina, M. S.; Rusanov, A. L.; Wagner, M.; Müllen, K. Macromolecules 2000, 33, 3525.

4. Centrone, A.; Brambilla, L.; Renouard, T.; Gherghel, L.; Mathis, C.; Müllen, K.; Zerbi, G. Carbon 2005, 43, 1593.

5. Rieger, R.; Müllen, K. J. Phys. Org. Chem. 2010, 23, 315.

6. Pinest, A.; Gibbyt, M. G.; Waugh, J. S. J. Chem. Phys. 1973, 59, 569.

7. Frye, J. S.; Maciel, G. E. J. Magn. Reson. 1982, 48, 125.

8. Morcombe, C. R.; Zilm, K. W. J. Magn. Reson. 2003, 162, 479.

9. Hayashi, S.; Hayamizu, K. Bull. Chem. Soc. Jpn. 1991, 64, 685.

10. Chen, Q.; Hou, S. S.; Schmidt-Rohr, K. Solid. State. Nucl. Mag. Reson. 2004, 26, 11.

11. Feike, M.; Demco, D. E.; Graf, R.; Gottwald, J.; Hafner, S. H. W. J. Magn. Reson. Ser. A 1996, 122, 214.

12. Saalwächter, K.; Lange, F.; Matyjaszewski, K.; Huang, C. F.; Graf, R. J. Magn. Reson. 2011, 212, 204.

13. Equbal, A.; Bjerring, M.; Madhu, P. K.; Nielsen, N. C. J. Chem. Phys. 2015, 142, 184201.

14. Zhang, Q.; Lin, W.; Chen, Q.; Yang, Guang. Macromolecules 2000, 33, 8904.

15. Brown, S. P.; Schnell, I.; Brand, J. D.; Mu1llen, K.; Spiess. H. W. J. Am. Chem. Soc. 1999, 121, 6712.

16. Ochsenfeld, C.; Brown, S. P.; Schnell, I.; Gauss, J.; Spiess, H. W. J. Am. Chem. Soc. 2001, 123, 2597.

17. Hansen, M. R.; Feng, X.; Macho, V.; Müllen, K.; Spiess, H. W.; Floudas, G. Phys. Rev. Lett. 2011, 107, 257801. 
18. Narita, A.; Verzhbitskiy, I. A.; Frederickx, W.; Mali, K. S.; Jensen, S. A.; Hansen, M. R.; Bonn, M.; Feyter, S. D.; Casiraghi, C.; Feng, X.; Müllen, K. ACS nano. 2014, 8,11622 .

19. Feike, M.; Demco, D. E.; Graf, R.; Gottwald, J.; Hafner, S.; Spiess, H. W. J. Magn. Reson. 1996, 122, 214.

20. Saalwächter, K.; Saalwächter, K,; Lange, F; Matyjaszewski, K.; Huang, C. F.; Graf, R. J. Magn. Reson. 2011, 212, 204.

21. Hyper Chem (TM) Professional 7.5 (Hypercube Inc., Gainesville, Florida). 